Publ. Mat. 51 (2007), 77-120

\title{
AN ACCURACY IMPROVEMENT IN EGOROV'S THEOREM
}

\author{
Jorge Drumond Silva
}

\begin{abstract}
We prove that the theorem of Egorov, on the canonical transformation of symbols of pseudodifferential operators conjugated by Fourier integral operators, can be sharpened. The main result is that the statement of Egorov's theorem remains true if, instead of just considering the principal symbols in $S^{m} / S^{m-1}$ for the pseudodifferential operators, one uses refined principal symbols in $S^{m} / S^{m-2}$, which for classical operators correspond simply to the principal plus the subprincipal symbol, and can generally be regarded as the first two terms of its Weyl symbol expansion: we call it the principal Weyl symbol of the pseudodifferential operator. Particular unitary Fourier integral operators, associated to the graph of the canonical transformation, have to be used in the conjugation for the higher accuracy to hold, leading to microlocal representations by oscillatory integrals with specific symbols that are given explicitly in terms of the generating function that locally describes the graph of the transformation. The motivation for the result is based on the optimal symplectic invariance properties of the Weyl correspondence in $\mathbb{R}^{n}$ and its symmetry for real symbols.
\end{abstract}

\section{Introduction}

1.1. General description of Egorov's theorem and of the improvement. From the perspective of microlocal analysis, and for now still not being very precise in the technical details of our description

2000 Mathematics Subject Classification. Primary: 58J40; Secondary: 47G30, 35S30. Key words. Egorov's theorem, conjugation by Fourier integral operators, canonical transformation of pseudodifferential operators, Weyl quantization, Weyl calculus.

This paper is based on the author's PhD thesis at Princeton University. The author is deeply indebted to his thesis advisor, Charles Fefferman, for the constant encouragement and invaluable guidance, suggestions and insights. To J. Sjöstrand a special acknowledgement is due for having pointed out his own work in a similar result. The author was partially supported by FCT through grant SFRH/BPD/1613/2000, program POCTI/FEDER and grant POCI/FEDER/MAT/55745/2004. 
(see the next section for that), the classical theorem of Egorov [Ego] establishes that the principal symbol

$$
\sigma(P) \in S^{m}\left(T^{*} X\right) / S^{m-1}\left(T^{*} X\right)
$$

of a pseudodifferential operator $P \in \Psi^{m}(X)$ is microlocally transformed into

$$
\sigma(Q)=\sigma(P) \circ \Phi,
$$

under a local homogeneous canonical transformation

$$
\Phi: \Gamma_{\left(y_{0}, \eta_{0}\right)} \subset T^{*} Y \backslash 0 \rightarrow \Gamma_{\left(x_{0}, \xi_{0}\right)} \subset T^{*} X \backslash 0,
$$

when $Q \in \Psi^{m}(Y)$ is obtained from the conjugation of $P$ by any elliptic Fourier integral operator $A \in I^{\mu}\left(X \times Y, C_{\Phi}\right)$, associated to the graph $C_{\Phi} \subset\left(T^{*} X \backslash 0\right) \times\left(T^{*} Y \backslash 0\right)$ of $\Phi$,

$$
Q=A^{-1} P A,
$$

where $A^{-1} \in I^{-\mu}\left(Y \times X, C_{\Phi^{-1}}\right)$ is a microlocal parametrix for $A$. The theorem can also be stated in a global form, with a full symplectomorphism from the whole of $T^{*} Y \backslash 0$ onto $T^{*} X \backslash 0$ and using global elliptic Fourier integral operators associated to it. But the most general case is just microlocal, with transformations occurring between small open conic neighborhoods $\Gamma_{\left(y_{0}, \eta_{0}\right)}$ and $\Gamma_{\left(x_{0}, \xi_{0}\right)}$ of points $\left(y_{0}, \eta_{0}\right) \in T^{*} Y \backslash 0$ and $\left(x_{0}, \xi_{0}\right)=\Phi\left(y_{0}, \eta_{0}\right) \in T^{*} X \backslash 0$, respectively.

The central result in this article is an accuracy improvement of this classical version. The setting of the theorem remains the same and by improved accuracy we mean that the microlocal canonical transformation of the pseudodifferential operator holds, not just for the usual principal symbol in $S^{m} / S^{m-1}$, but for a more refined symbol in $S^{m} / S^{m-2}$, thus extending the result to lower order terms. We show that this higher accuracy can be achieved if $P \in \Psi^{m}(X)$ is conjugated by a specific microlocally unitary Fourier integral operator, associated to the canonical transformation, instead of a general elliptic one as described above for the usual version of the theorem. An elliptic Fourier integral operator $A \in I^{0}\left(X \times Y, C_{\Phi}\right)$ is said to be microlocally unitary if its adjoint is a microlocal parametrix, i.e., if

$$
A^{*} A-I_{Y} \in \Psi^{-\infty} \text { and } A A^{*}-I_{X} \in \Psi^{-\infty} \text {, }
$$

in conic neighborhoods of corresponding points (mapped to each other by $\Phi)$ of the cotangent bundles $T^{*} Y \backslash 0$ and $T^{*} X \backslash 0$, respectively. Actually, the degree of accuracy in the statement of our main theorem only requires this unitary property to hold modulo operators in $\Psi^{-2}$.

To make this first description of the result clearer, let us fix coordinates on $X$ and $Y$, and assume that the complete symbol of $P \in \Psi^{m}(X)$ 
(in any coordinates) is classical, i.e., given by an asymptotic expansion of positively homogeneous symbols of decreasing integer degree

$$
p(x, \xi) \sim p_{m}(x, \xi)+p_{m-1}(x, \xi)+\cdots,
$$

with

$$
p_{j}(x, \lambda \xi)=\lambda^{j} p_{j}(x, \xi), \quad \text { for } \quad \lambda>0, \quad j=m, m-1, m-2, \ldots
$$

The improved result then asserts that there exists an appropriate unitary operator $A$ such that the pseudodifferential operator $Q \in \Psi^{m}(Y)$ obtained from the conjugation of $P$ by it,

$$
Q=A^{*} P A,
$$

is also given by a classical symbol

$$
q(y, \eta) \sim q_{m}(y, \eta)+q_{m-1}(y, \eta)+\cdots
$$

that satisfies not only

$$
q_{m}(y, \eta)=p_{m}(\Phi(y, \eta))
$$

microlocally, at the level of the principal symbol - the homogeneous term of highest degree in the expansion - like in the usual theorem, but also down to the subprincipal symbol, at the next level of homogeneity and accuracy. This means that one also gets

$$
\operatorname{Sub}(Q)(y, \eta)=\operatorname{Sub}(P)(\Phi(y, \eta)),
$$

where the subprincipal symbol of a classical operator

$$
\operatorname{Sub}(P)(x, \xi)=p_{m-1}(x, \xi)+\frac{i}{2} \sum_{k=1}^{n} \partial_{x_{k}} \partial_{\xi_{k}} p_{m}(x, \xi),
$$

is homogeneous of degree $m-1$. Therefore, the sum of the principal and the subprincipal symbols of $P$,

(3) $p_{m}(x, \xi)+\operatorname{Sub}(P)(x, \xi)=p_{m}(x, \xi)+p_{m-1}(x, \xi)+\frac{i}{2} \sum_{k=1}^{n} \partial_{x_{k}} \partial_{\xi_{k}} p_{m}(x, \xi)$,

is the refined symbol in $S^{m} / S^{m-2}$, all of which gets microlocally transformed by the canonical transformation according to (1) and (2), as a consequence of the conjugation.

We will exhibit the explicit formula of an oscillatory integral that represents, in local coordinates, the unitary Fourier integral operator adequate to achieve the higher accuracy. As a matter of fact, the proof of the main result relies on computations using that explicit formula. This oscillatory integral will be written in terms of the generating function 
of the canonical transformation, which is always well defined locally, as long as appropriate coordinates are chosen on $Y$.

It is clear that to be able to state the theorem in coordinate invariant terms, the subprincipal symbol of a pseudodifferential operator should also be invariantly defined as a function on the cotangent bundle, just as the principal symbol is. But the invariance of (3) is ensured precisely by a particular case of this more accurate version of Egorov's theorem which has actually been well known for a long time. That is when the unitary Fourier integral operator $A$ is the pull-back $\kappa^{*}$ of halfdensities $u \in C^{\infty}\left(Y, \Omega_{Y}^{1 / 2}\right)$ under a diffeomorphism $\kappa: X \rightarrow Y$. Then $A: C^{\infty}\left(Y, \Omega_{Y}^{1 / 2}\right) \rightarrow C^{\infty}\left(X, \Omega_{X}^{1 / 2}\right)$ is given in local coordinates by

$$
u(y) \mapsto A u(x)=\left(\kappa^{*} u\right)(x)=\left|\operatorname{det} \kappa^{\prime}(x)\right|^{1 / 2} u(\kappa(x)),
$$

so that its adjoint $A^{*}: C^{\infty}\left(X, \Omega_{X}^{1 / 2}\right) \rightarrow C^{\infty}\left(Y, \Omega_{Y}^{1 / 2}\right)$

$$
v(x) \mapsto A^{*} v(y)=\left|\operatorname{det}\left(\kappa^{-1}\right)^{\prime}(y)\right|^{1 / 2} v\left(\kappa^{-1}(y)\right),
$$

is simply the inverse pull-back $A^{*}=\left(\kappa^{-1}\right)^{*}=\left(\kappa^{*}\right)^{-1}=A^{-1}$, thus showing that $A$ is unitary. When $u \in C_{c}^{\infty}\left(Y, \Omega_{Y}^{1 / 2}\right)$ is compactly supported, with the support contained in a coordinate neighborhood of $Y,(4)$ is obviously given by the more familiar local representation of a Fourier integral operator, as an oscillatory integral,

$$
A u(x)=\frac{1}{(2 \pi)^{n}} \int e^{i \kappa(x) \cdot \eta}\left|\operatorname{det} \kappa^{\prime}(x)\right|^{1 / 2} \hat{u}(\eta) d \eta,
$$

to which corresponds the Schwartz kernel

$$
K_{A}(x, y)=\frac{1}{(2 \pi)^{n}} \int e^{i(\kappa(x)-y) \cdot \eta}\left|\operatorname{det} \kappa^{\prime}(x)\right|^{1 / 2} d \eta,
$$

where $n$ is the dimension both of $Y$ and $X$, and $\hat{u}$ is the Fourier transform of $u$. Observe that, from a coordinate invariant point of view, the kernel (6) is a distribution half-density on $X \times Y$, i.e. $K_{A} \in \mathcal{D}^{\prime}(X \times$ $\left.Y, \Omega_{X \times Y}^{1 / 2}\right)$, just like they are normally considered to be in the global theory of Fourier integral operators. The conjugation

$$
u \mapsto A^{*} P A u=\left(\kappa^{-1}\right)^{*} P\left(\kappa^{*} u\right),
$$

therefore can be interpreted as the rule for a change of coordinates of a pseudodifferential operator acting on half-densities and it is in this context that the result is normally presented ([Dui $],[\mathbf{G r S j}],[\mathbf{H o ̈ r} \mathbf{3}])$. The usually stated conclusion, that can be proved easily by explicit computations using the local representations of the operators, is that both the principal as well as the subprincipal symbols, of such pseudodifferential 
operators acting on half-densities (when given by classical symbols), are invariantly defined as functions on the cotangent bundle. This is a consequence of properties (1) and (2) being satisfied where, in this case, the (global) canonical transformation

$$
\Phi_{\kappa}: T^{*} Y \backslash 0 \rightarrow T^{*} X \backslash 0,
$$

is the canonical change of coordinates of the covectors for a diffeomorphism of the base manifold

$$
(x, \xi)=\Phi_{\kappa}(y, \eta)=\left(\kappa^{-1}(y),{ }^{t} \kappa^{\prime}(x) \eta\right) .
$$

It is homogeneous of degree one, in the fibers, and its generating function, also global and homogeneous in $\eta$, is the phase in (5),

$$
S(x, \eta)=\kappa(x) \cdot \eta .
$$

These pieces put together mean that (5), or (6), represents a unitary Fourier integral operator $A \in I^{0}\left(X \times Y, C_{\Phi_{\kappa}} ; \Omega_{X \times Y}^{1 / 2}\right)$, of order 0 , from half-densities on $Y$ to half-densities on $X$, associated to the graph of $\Phi_{\kappa}$. And that conjugating by $A$ any pseudodifferential operator $P \in$ $\Psi^{m}\left(X, \Omega_{X}^{1 / 2}\right)$, acting on half-densities on $X$, according to (7), produces the equivalent pseudodifferential operator acting on half-densities on $Y$, with a more accurate coordinate transformation law for their symbols than the usual one, which can be regarded as a particular case of accuracy improvement in Egorov's theorem. It is important to point out that the symbol of order 0 , in (5) or (6), is related to the generating function of the canonical transformation, by

$$
\left|\operatorname{det} \kappa^{\prime}(x)\right|^{1 / 2}=\left|\operatorname{det} \frac{\partial^{2} S}{\partial x \partial \eta}(x, \eta)\right|^{1 / 2} .
$$

So, in exactly the same way as the principal symbol of a general pseudodifferential operator acting on functions is defined invariantly as an equivalence class in $S^{m}\left(T^{*} X\right) / S^{m-1}\left(T^{*} X\right)$, this result now allows the definition of a more accurate invariant principal symbol for general pseudodifferential operators acting on half-densities (L. Hörmander calls it simply a refined principal symbol, in [Hör3]), as an equivalence class in $S^{m}\left(T^{*} X\right) / S^{m-2}\left(T^{*} X\right)$. If $p(x, \xi) \in S^{m}$ is the complete symbol of the pseudodifferential operator $P \in \Psi^{m}\left(X, \Omega_{X}^{1 / 2}\right)$, in any local system of coordinates, then this more accurate invariant symbol is given in these coordinates by

$$
p(x, \xi)+\frac{i}{2} \sum_{k=1}^{n} \partial_{x_{k}} \partial_{\xi_{k}} p(x, \xi) \quad\left(\bmod S^{m-2}\right) .
$$


In the particular case of operators with classical symbols this is the sum of the principal and subprincipal symbols, seen above in (3). This also yields the sharper isomorphism

$$
\Psi^{m}\left(X, \Omega_{X}^{1 / 2}\right) / \Psi^{m-2}\left(X, \Omega_{X}^{1 / 2}\right) \cong S^{m}\left(T^{*} X\right) / S^{m-2}\left(T^{*} X\right),
$$

analogous to the one for the usual principal symbol. The geometric framework of having the operators acting on half-densities is crucial for the result to hold here, for only then is the change of coordinates a unitary operator. That is something which does not happen in the more common rule for a change of coordinates for operators acting on functions, where the invariance can only be established for the principal symbol. This particular example, including the geometric setting, the oscillatory integral (5) and its symbol's dependence on the generating function of the canonical transformation (8), already contains all the ingredients of the general improvement of Egorov's theorem, that we present in this paper.

It is a well known fact that, from the point of view of the Weyl quantization, this refined principal symbol of a pseudodifferential operator can be very naturally identified with the first two terms of the asymptotic expansion of its Weyl symbol. This interpretation is quite important because the properties of the Weyl calculus in $\mathbb{R}^{n}$ provide the essential motivation for the setting in which one should expect the improved accuracy in Egorov's theorem to hold. Section 2, of this article, is precisely devoted to bringing up the parallelism between the properties of the Weyl calculus, particularly those that concern symplectic invariance and symmetry, and this improvement of Egorov's theorem in a microlocal analysis setting. Because of the fundamental role of the Weyl quantization, underlying this result, we will call (9) the principal Weyl symbol of a pseudodifferential operator.

A relevant final observation is that, in order to state the theorem within a geometric framework that is coordinate invariant, we will consider all the operators to be acting on half-densities, just like in the particular case of the change of coordinates mentioned above. On the one hand, this should really be the natural setting, for when one deals with the coordinate invariant global theory of Fourier integral operators ([Dui], [Hör4], [Tre]), their Schwartz kernels are normally considered to be Fourier integral distribution half-densities on the product space, therefore leading to operators acting on half-densities from one base manifold to the other. So, if a pseudodifferential operator is going to be conjugated by these Fourier integral operators, it really has to act on half-densities too for the composition of operators to be well defined. 
Which, as we have just recalled, is precisely the requirement for their principal Weyl symbols to be invariantly defined on the cotangent bundle, making their composition with canonical transformations between such bundles completely coherent. On the other hand, as pointed out in the previous paragraph, our main motivation comes from the properties of the Weyl quantization on $\mathbb{R}^{n}$, with the $L^{2}\left(\mathbb{R}^{n}\right)$ inner product structure. So if we want to have coordinate invariant analogues of self-adjoint and unitary operators, for example, but now on general manifolds without a distinguished positive density which allows the identification of functions and densities, we need to think of the natural $L^{2}$ space structure on manifolds: again the same half-densities and their coordinate invariant inner product.

Everything thus fits together to state the result, independently of coordinates: given a local homogeneous symplectomorphism $\Phi$, from an open conic neighborhood in $T^{*} Y \backslash 0$ onto one in $T^{*} X \backslash 0$, there is an appropriate unitary Fourier integral operator of order zero associated to it $A \in I^{0}\left(X \times Y, C_{\Phi} ; \Omega_{X \times Y}^{1 / 2}\right)$ such that, conjugating any pseudodifferential operator $P \in \Psi^{m}\left(X, \Omega_{X}^{1 / 2}\right)$, acting on half-densities, by $A$ yields another pseudodifferential operator $Q=A^{*} P A \in \Psi^{m}\left(Y, \Omega_{Y}^{1 / 2}\right)$ whose principal Weyl symbol, invariantly defined on $T^{*} Y$, is microlocally equal to $P$ 's composed with $\Phi$.

It is interesting to point out that analogous results, both on the invariance of (9) under changes of coordinates, as well as the corresponding improved form of Egorov's theorem (the former being really a particular case of the latter), were already well known for classical pseudodifferential operators acting on functions, when restricted to points where the principal symbol vanishes of second order (see, for example, Chapter XV, $\S 2$ in [Tay]). This subset of the cotangent bundle

$$
\Sigma_{2}=\left\{(x, \xi) \in T^{*} X \backslash 0: p_{m}(x, \xi)=0 \text { and } \partial_{x, \xi} p_{m}(x, \xi)=0\right\},
$$

is the set of double characteristics of $P \in \Psi^{m}(X)$, with symbol $p(x, \xi) \sim$ $p_{m}(x, \xi)+p_{m-1}(x, \xi)+\cdots$. At these points, of course, $(9)$ reduces to the subprincipal symbol. And in fact, besides the Weyl calculus, the study of multiple characteristics of operators is the other instance where the subprincipal symbol naturally occurs and plays a very important role. These theorems then state that the subprincipal symbol of a classical operator (acting on functions) is invariantly defined on $\Sigma_{2}$ and that its restriction to this set is canonically transformed if $P$ is conjugated by any elliptic Fourier integral operator associated to the canonical transformation. Of course the result that we present in this article shows that 
the appropriate geometrical framework that allows for the full canonical transformation of both the principal as well as the subprincipal symbols, without any restrictions, is really to consider all the operators acting on half-densities and to conjugate by appropriate unitary Fourier integral operators. These theorems then show that, for operators acting on functions, the setting that somehow reproduces the more accurate form of Egorov's theorem, but still within the general framework of the classical version, is to restrict to those points where the principal symbol of the operator has zeros of second order and for which the principal Weyl symbol (9) becomes just the subprincipal one.

Much closer to our result in this paper, and to the author's unawareness while working on this subject for his $\mathrm{PhD}$ thesis, B. Helffer and J. Sjöstrand in $[\mathbf{H e S j}]$, and M. Hitrik and J. Sjöstrand in $[\mathbf{H i S j}]$ proved the same type of result, in the framework of semi-classical analysis and with Weyl operators. In $[\mathbf{H e S j}]$ the authors prove the existence of unitary Fourier integral operators that yield the improved accuracy version of Egorov's theorem, when used in the conjugation of Weyl operators. Pursuing a question that was left open in the previous paper, in $[\mathbf{H i S j}]$ a necessary and sufficient condition is then presented that characterizes the symbols of such Fourier integral operators. Even though the motivation for our result also had its origin in the properties of the Weyl quantization, it differs from the one in these articles in that our statement is made exclusively within the framework of classical microlocal analysis, in coordinate invariant terms. Therefore, we emphasize the particular geometrical context that enables the reproduction of the analogous properties of the Weyl operator in $\mathbb{R}^{n}$, but in our case for pseudodifferential operators defined on general manifolds. Despite being longer, our method of proof is also different and simply consists, nevertheless, in a straightforward computation using the well known asymptotic expansion formulas derived from the method of stationary phase.

1.2. Precise statement of the result. We now proceed to state the main result in this article, in rigorous terms. We firstly recall the classical version of Egorov's theorem in order to carefully review its hypotheses and see how they come together to produce the known result. Based on that analysis, we then point out exactly the specific conditions that, comparatively, have to be imposed to achieve the improved accuracy. Considering what was described in the previous section, it is clear that a special focus has to be placed on the particular unitary Fourier integral operator used in the conjugation, its local representation as an oscillatory integral and the corresponding symbol. 
Starting with the geometrical framework, let $X$ and $Y$ be two $C^{\infty}$ manifolds of the same dimension $n$ and let

$$
\Phi: \Gamma_{\left(y_{0}, \eta_{0}\right)} \subset T^{*} Y \backslash 0 \rightarrow \Gamma_{\left(x_{0}, \xi_{0}\right)} \subset T^{*} X \backslash 0
$$

be a homogeneous symplectomorphism, from an open conic neighborhood $\Gamma_{\left(y_{0}, \eta_{0}\right)}$ of $\left(y_{0}, \eta_{0}\right) \in T^{*} Y \backslash 0$ onto an open conic neighborhood $\Gamma_{\left(x_{0}, \xi_{0}\right)}$ of $\left(x_{0}, \xi_{0}\right)=\Phi\left(y_{0}, \eta_{0}\right) \in T^{*} X \backslash 0$. As usual, this means that $\Phi: \Gamma_{\left(y_{0}, \eta_{0}\right)} \rightarrow \Gamma_{\left(x_{0}, \xi_{0}\right)}$ is a bijective canonical transformation with respect to the canonical symplectic forms on the cotangent bundles, i.e.,

$$
\Phi^{*} \sigma_{X}=\sigma_{Y}
$$

(in local coordinates $\sigma_{X}=\sum_{i=1}^{n} d \xi_{i} \wedge d x_{i}$ and $\sigma_{Y}=\sum_{i=1}^{n} d \eta_{i} \wedge d y_{i}$ ) which is homogeneous of degree one in the fibers. Equivalently, the graph of $\Phi$,

$C_{\Phi}=\left\{(x, \xi, y, \eta) \in \Gamma_{\left(x_{0}, \xi_{0}\right)} \times \Gamma_{\left(y_{0}, \eta_{0}\right)}:(x, \xi)=\Phi(y, \eta)\right\} \subset\left(T^{*} X \backslash 0\right) \times\left(T^{*} Y \backslash 0\right)$, is a conic Lagrangian submanifold of $T^{*}(X \times Y) \backslash 0$ with respect to the symplectic form $\pi_{X}^{*} \sigma_{X}-\pi_{Y}^{*} \sigma_{Y}{ }^{1}$.

In the classical version of the theorem, the Fourier integral operator used in the conjugation can be any elliptic one at $\left(x_{0}, \xi_{0}, y_{0}, \eta_{0}\right) \in C_{\Phi}$, associated to the graph of the symplectomorphism. So let $A$ be a properly supported Fourier integral operator from half-densities on $Y$ to halfdensities on $X$,

$$
A: C_{c}^{\infty}\left(Y, \Omega_{Y}^{1 / 2}\right) \rightarrow C_{c}^{\infty}\left(X, \Omega_{X}^{1 / 2}\right) \quad\left(\text { also for } C^{\infty}, \mathcal{E}^{\prime}, \mathcal{D}^{\prime}\right),
$$

with Schwartz kernel $K_{A} \in I^{\mu}\left(X \times Y, C_{\Phi}^{\prime} ; \Omega_{X \times Y}^{1 / 2}\right)$, a Fourier integral distribution half-density on $X \times Y$, of order $\mu \in \mathbb{R}$, associated to the graph $C_{\Phi} \subset\left(T^{*} X \backslash 0\right) \times\left(T^{*} Y \backslash 0\right)$ of $\Phi$ : the wavefront set of $K_{A}$ satisfies

$$
W F\left(K_{A}\right) \subset C_{\Phi}^{\prime}=\left\{(x, \xi, y, \eta) \in T^{*}(X \times Y) \backslash 0:(x, \xi, y,-\eta) \in C_{\Phi}\right\} .
$$

We are using the usual notation of microlocal analysis where the prime on $C_{\phi}^{\prime}$ means the twisted canonical relation (actually, in this case, the twisted graph of a canonical transformation) in $T^{*}(X \times Y) \backslash 0$. Therefore $C_{\Phi}^{\prime}$ is a Lagrangian submanifold of $T^{*}(X \times Y) \backslash 0$ with respect to the canonical symplectic form on this bundle, $\sigma_{X \times Y} \cong \pi_{X}^{*} \sigma_{X}+\pi_{Y}^{*} \sigma_{Y}$.

Based on the existence of parametrices for elliptic pseudodifferential operators, if $A$ is elliptic at $\left(x_{0}, \xi_{0}, y_{0}, \eta_{0}\right) \in C_{\Phi}$ it is easy to construct ([Dui],$[\mathbf{G r S j}]$, [Tre]) a microlocal parametrix for it: a properly

\footnotetext{
${ }^{1} \pi_{X}$ and $\pi_{Y}$ are the canonical projections from the product bundle $T^{*}(X \times Y) \cong$ $T^{*} X \times T^{*} Y$ onto the components $T^{*} X$ and $T^{*} Y$, respectively.
} 
supported Fourier integral operator $B \in I^{-\mu}\left(Y \times X, C_{\Phi^{-1}} ; \Omega_{Y \times X}^{1 / 2}\right)^{2}$, such that

$$
B A-I_{Y} \in \Psi^{-\infty}\left(V_{Y}\right) \text { and } A B-I_{X} \in \Psi^{-\infty}\left(V_{X}\right),
$$

in open conic neighborhoods $V_{Y} \subset T^{*} Y \backslash 0$, of $\left(y_{0}, \eta_{0}\right)$, and $V_{X} \subset T^{*} X \backslash 0$, of $\left(x_{0}, \xi_{0}\right)$.

With all this setup, Egorov's theorem then states ([BFG], $[\mathbf{G r S j}]$, [Hör4], [Tre]) that, given any properly supported pseudodifferential operator of order $m \in \mathbb{R}$, acting on half-densities on $X$,

$$
P \in \Psi^{m}\left(X, \Omega_{X}^{1 / 2}\right)
$$

when conjugating it by the Fourier integral operator $A$, with its microlocal parametrix $B$,

$$
Q=B P A,
$$

one obtains a pseudodifferential operator $Q \in \Psi^{m}\left(Y, \Omega_{Y}^{1 / 2}\right)$ of the same order $m$, acting on half-densities on $Y$. The principal symbols, $\sigma(P) \in$ $S^{m}\left(T^{*} X\right) / S^{m-1}\left(T^{*} X\right)$ and $\sigma(Q) \in S^{m}\left(T^{*} Y\right) / S^{m-1}\left(T^{*} Y\right)$, satisfy

$$
\sigma(Q)=\sigma(P) \circ \Phi
$$

in a conic neighborhood of $\left(y_{0}, \eta_{0}\right) \in T^{*} Y \backslash 0$.

\section{Remarks.}

i) As mentioned in the previous section, a global version of the theorem is equally possible. To begin with, the canonical transformation has to be a global symplectomorphism, from the whole of $T^{*} Y \backslash 0$ onto $T^{*} X \backslash 0$, whose graph $C_{\Phi} \subset\left(T^{*} X \backslash 0\right) \times\left(T^{*} Y \backslash 0\right)$ is a closed homogeneous Lagrangian submanifold of $T^{*}(X \times Y) \backslash 0$. For the conjugation, one then considers a global elliptic Fourier integral operator associated to $C_{\Phi}$, i.e., such that the (twisted) wavefront set of its Schwartz kernel coincides with the whole graph and is elliptic at every one of its points. Global, properly supported, parametrices are as easy to construct as for the microlocal case, starting with an

\footnotetext{
${ }^{2}$ There is usually no standard difference in notation to distinguish a class of Fourier integral operators from the class of Fourier integral distributions that forms the set of their corresponding Schwartz kernels. Exactly the same notation is used indistinguishably for both, for example in [Hör4]. Here, for the kernels of Fourier integral operators we follow the usual notation that is used for classes of Fourier integral distributions, indicating the twisted canonical graph $C_{\Phi}^{\prime}$ as the Lagrangian submanifold of $T^{*}(X \times Y) \backslash 0$ (with respect to the canonical symplectic form $\sigma_{X \times Y}=\sigma_{X}+\sigma_{Y}$ ) in which the wavefront set of the kernels is contained. Whereas, for the classes of operators themselves the only difference is that we write the actual graph $C_{\Phi}$, and not its twisted form $C_{\Phi}^{\prime}$, as the relevant object.
} 
elliptic Fourier integral operator associated to the inverse graph (the adjoint operator, for example) and then using the parametrix of the elliptic pseudodifferential operator resulting from the composition of the two, to complete the operator inversion: this procedure is quite standard. The final statement is exactly the same as above, except that (12) becomes valid for the whole cotangent bundle $T^{*} Y \backslash 0$. The argument can, nevertheless, be reduced to the microlocal case by using locally finite partitions of unity on the cosphere bundle of $X \times Y$ to break or patch up those global operators from microlocal representations in conic neighborhoods of the points of the graph $C_{\phi}$. So, in this sense, the microlocal version of Egorov's theorem is the most general.

ii) The proof of Egorov's theorem becomes very simple and computations involving the microlocal representations by oscillatory integrals can be avoided, if one falls back on the theorem about composition of Fourier integral operators ([Dui], [GrSj], [Hör4], [Tre]) (which, in that case, is where the computations with oscillatory integrals take place). When the (properly supported) Fourier integral operators are associated, not to general homogeneous canonical relations, but to graphs of homogeneous symplectomorphisms, this theorem ensures that their composition yields another Fourier integral operator associated to the graph of the composed symplectomorphisms and, just like it happens in the particular case of the composition of pseudodifferential operators, here too the final principal symbol of the composition is simply the product of the intervening principal symbols ([Hör4, Section 25.3]; [Tre, Chapter VIII, Section 6]). For this to hold, one needs to consider the invariant definition of the principal symbol of a Fourier integral operator associated to the graph of a canonical transformation, with the natural symplectic half-density factored out.

When $A \in I^{\mu}\left(X \times Y, C_{\Phi} ; \Omega_{X \times Y}^{1 / 2}\right)$ its principal symbol is an equivalence class of sections of $\Omega_{C_{\Phi}}^{1 / 2} \otimes \mathcal{L}_{C_{\Phi}}$ over $C_{\Phi}$, in the quotient

$$
S^{\mu+\frac{n}{2}}\left(C_{\Phi}, \Omega_{C_{\Phi}}^{1 / 2} \otimes \mathcal{L}_{C_{\Phi}}\right) / S^{\mu+\frac{n}{2}-1}\left(C_{\Phi}, \Omega_{C_{\Phi}}^{1 / 2} \otimes \mathcal{L}_{C_{\Phi}}\right),
$$

where $\Omega_{C_{\Phi}}^{1 / 2}$ is the half-density and $\mathcal{L}_{C_{\Phi}}$ the Keller-Maslov-Hörmander line bundle, both on $C_{\Phi}$. On graphs of canonical transformations there is a preferred positive density, obtained by the projection pullback $\pi_{X}^{*}$ of the canonical (symplectic) density from $T^{*} X$ (in local coordinates $|d x||d \xi|)$. And because $C_{\Phi}$ is a Lagrangian submanifold of $T^{*}(X \times Y) \backslash 0$ with respect to the symplectic form $\pi_{X}^{*} \sigma_{X}-\pi_{Y}^{*} \sigma_{Y}$, 
then $\pi_{X}^{*} \sigma_{X}=\pi_{Y}^{*} \sigma_{Y}$ on $C_{\Phi}$, so that the preferred density can also be obtained by the pull-back $\pi_{Y}^{*}$ from $T^{*} Y$ (in local coordinates $|d y||d \eta|)$. It is very convenient, and common, to factor out the half-densities from the principal symbol in (13) by using this preferred density. So, a more natural invariant principal symbol is obtained for Fourier integral operators associated to graphs of canonical transformations, by dividing the one in (13) by the halfdensity $|d x|^{1 / 2}|d \xi|^{1 / 2}=|d y|^{1 / 2}|d \eta|^{1 / 2}$ on $C_{\Phi}$,

$$
\sigma(A) \in S^{\mu}\left(C_{\Phi}, \mathcal{L}_{C_{\Phi}}\right) / S^{\mu-1}\left(C_{\Phi}, \mathcal{L}_{C_{\Phi}}\right) .
$$

This is the principal symbol that should be used in the product rule, mentioned above, when composing Fourier integral operators associated to canonical transformations. And it coincides with the principal symbol of a pseudodifferential operator, when the symplectomorphism $\Phi$ is just the identity and the Keller-Maslov-Hörmander line bundle is trivialized by the usual non-degenerate phase.

Now, the statement of the classical version of Egorov's theorem, as above, only concerns principal symbols and all the Fourier integral operators involved, including the pseudodifferential operator, are associated to graphs of canonical transformations. The conjugation (11) then yields a Fourier integral operator, of order $-\mu+m+$ $\mu=m$, associated to the identity symplectomorphism on $T^{*} Y \backslash 0$. Therefore, $Q$ is a pseudodifferential operator on $Y$ of the same order as $P$. And the resulting principal symbol is simply the product of the principal symbols of $B, P$ and $A$ :

$$
\sigma(Q)_{{ }_{(y, \eta)}}=\left.\left.\left.\sigma(B)\right|_{(y, \eta, \Phi(y, \eta))} \sigma(P)\right|_{\Phi(y, \eta)} \sigma(A)\right|_{(\Phi(y, \eta), y, \eta)} .
$$

When $B$ is a microlocal parametrix of $A$, its principal symbols are the inverse of each other in a conic neighborhood of $\left(x_{0}, \xi_{0}, y_{0}, \eta_{0}\right)$, so (12) follows from (15).

iii) In view of the previous remark, particularly from (15), it is clear that it is not even required that the operator $B$, in (11), be a full parametrix of $A$ for (12) to hold. In fact, the only thing that is needed is for its principal symbol

$$
\sigma(B) \in S^{-\mu}\left(C_{\Phi^{-1}}, \mathcal{L}_{C_{\Phi^{-1}}}\right) / S^{-\mu-1}\left(C_{\Phi^{-1}}, \mathcal{L}_{C_{\Phi^{-1}}}\right),
$$

to be an inverse of (14) in a conic neighborhood of $\left(y_{0}, \eta_{0}, x_{0}, \xi_{0}\right) \in$ $C_{\Phi^{-1}}$, i.e., the transformation (12) will still be obtained if only

$$
\left.\sigma(B)_{(y, \eta, \Phi(y, \eta))} \sigma(A)\right|_{(\Phi(y, \eta), y, \eta)} \equiv 1 \quad\left(\bmod S^{-1}\right),
$$


is satisfied in a conic neighborhood of $\left(y_{0}, \eta_{0}\right) \in T^{*} Y \backslash 0$. The existence of such an inverse for $\sigma(A)$ is exactly the definition of ellipticity of $A$ at $\left(x_{0}, \xi_{0}, y_{0}, \eta_{0}\right)$. Hence, any Fourier integral operator $B \in I^{-\mu}\left(Y \times X, C_{\Phi^{-1}} ; \Omega_{Y \times X}^{1 / 2}\right)$, of order $-\mu$, associated to the graph of $\Phi^{-1}$, whose principal symbol satisfies (16) will do the job.

iv) In applications, the goal is often just to transform the symbols of pseudodifferential operators microlocally. And in that case, conjugation by any chosen Fourier integral operator associated to the graph of the canonical transformation, which is elliptic at the point under consideration, will suffice to achieve the desired transformation. What is usually considered is an operator of order 0 , given explicitly in local coordinates by an oscillatory integral representation, with a non-degenerate phase based on the generating function of the canonical transformation $\Phi$ in a neighborhood of the point $\left(x_{0}, \xi_{0}, y_{0}, \eta_{0}\right)$, and with a symbol supported only sufficiently close.

Local coordinates can always be chosen on the manifold $Y$, around the point $y_{0}$, inducing the canonical coordinates on the cotangent bundle $T^{*} Y \backslash 0$, such that the $x, \eta$ projection of the graph

$$
C_{\Phi} \ni(x, \xi, y, \eta) \mapsto(x, \eta) \in \mathbb{R}_{x}^{n} \times\left(\mathbb{R}_{\eta}^{n} \backslash 0\right)
$$

is a local diffeomorphism, from an open conic neighborhood of $\left(x_{0}, \xi_{0}, y_{0}, \eta_{0}\right)$ on $C_{\Phi}$ onto one of $\left(x_{0}, \eta_{0}\right)$ (see Proposition 25.3.3 in $[\mathbf{H o ̈ r} \mathbf{3}]$, or Chapter 8 , in $[\mathbf{G r S j}]$ ). The choice of local coordinates on $X$ can be any one, here. Because $C_{\Phi}$ is a conic Lagrangian submanifold of $T^{*}(X \times Y) \backslash 0$ with respect to the symplectic form $\sigma_{X}-\sigma_{Y}=\sum_{j} d \xi_{j} \wedge d x_{j}-\sum d \eta_{j} \wedge d y_{j}$, there exists then a generating function $S$, defined in a (possibly smaller) open conic neighborhood of $\left(x_{0}, \eta_{0}\right)$, homogeneous of degree one in the $\eta$ variable, such that the corresponding points of the graph are parametrized by $S(x, \eta)$ according to

$$
(x, \xi, y, \eta) \in C_{\Phi} \quad \Longleftrightarrow \quad \xi=\frac{\partial S}{\partial x}(x, \eta) \quad \text { and } \quad y=\frac{\partial S}{\partial \eta}(x, \eta) .
$$

The phase

$$
\varphi(x, y, \eta)=S(x, \eta)-y \cdot \eta,
$$

is non-degenerate and defines $C_{\Phi}^{\prime}$ in the conic neighborhood of $\left(x_{0}, \xi_{0}, y_{0},-\eta_{0}\right)$, corresponding to the same points of the graph parametrized by $S(x, \eta)$. So if a symbol $a \in S^{0}\left(\mathbb{R}_{x, y}^{2 n} \times \mathbb{R}_{\eta}^{n}\right)$ is chosen, with support in a small enough compactly generated conic neighborhood of $\left(x_{0}, y_{0}, \eta_{0}\right)=\left(x_{0}, \partial_{\eta} S\left(x_{0}, \eta_{0}\right), \eta_{0}\right) \in \mathbb{R}_{x, y}^{2 n} \times\left(\mathbb{R}_{\eta}^{n} \backslash 0\right)$ so 
that it is contained in the domain of definition of the phase (18), then the oscillatory integral

$$
K_{A}(x, y)=\frac{1}{(2 \pi)^{n}} \int e^{i(S(x, \eta)-y \cdot \eta)} a(x, y, \eta) d \eta,
$$

defines the Schwartz kernel of a properly supported (in fact, compactly supported) Fourier integral operator $A \in I^{0}\left(X \times Y, C_{\Phi} ; \Omega_{X \times Y}^{1 / 2}\right)$, of order 0 , associated to the graph of $\Phi$. Its wavefront set satisfies $W F^{\prime}\left(K_{A}\right) \subset C_{\Phi}$ and more precisely is contained in the conic neighborhood of $\left(x_{0}, \xi_{0}, y_{0}, \eta_{0}\right)$ parametrized by the generating function. The invariant principal symbol $\sigma(A) \in S^{0}\left(C_{\Phi}, \mathcal{L}_{C_{\Phi}}\right) / S^{-1}\left(C_{\Phi}, \mathcal{L}_{C_{\Phi}}\right)$ of (19), with the natural half-density factored out as mentioned in Remark ii) and with the trivialization of the Keller-MaslovHörmander line bundle associated to the phase (18), is given by

$$
\begin{aligned}
& \sigma(A)_{\mid(\Phi(y, \eta), y, \eta)=\left(x, \partial_{x} S(x, \eta), \partial_{\eta} S(x, \eta), \eta\right)} \\
& \equiv \frac{a\left(x, \partial_{\eta} S(x, \eta), \eta\right)}{\left|\operatorname{det} \frac{\partial^{2} S}{\partial x \partial \eta}(x, \eta)\right|^{1 / 2}} \quad\left(\bmod S^{-1}\right),
\end{aligned}
$$

using $(x, \eta)$ as parameters on $C_{\Phi}([\mathbf{H o ̈ r} 4]$, [Tre]). Recall that the generating function of a canonical transformation satisfies $\operatorname{det} \frac{\partial^{2} S}{\partial x \partial \eta}(x, \eta) \neq 0$ in its conic domain of definition and that this determinant is a homogeneous function of degree zero, in the $\eta$ variable. Finally, one just has to be able to invert (20) in a conic neighborhood of $\left(x_{0}, \xi_{0}, y_{0}, \eta_{0}\right)$, for large $\eta$. This ellipticity of $A$ is ensured by picking a symbol $a \in S^{0}\left(\mathbb{R}_{x, y}^{2 n} \times \mathbb{R}_{\eta}^{n}\right)$ that also satisfies,

$$
\exists_{C>0} \quad|a(x, y, \eta)| \geq 1 / C, \quad \text { for }|\eta| \geq C,
$$

in an open conic neighborhood of $\left(x_{0}, y_{0}, \eta_{0}\right) \in \mathbb{R}_{x, y}^{2 n} \times\left(\mathbb{R}_{\eta}^{n} \backslash 0\right)$. A natural way to construct such a symbol is to start with a bump function, on the surface of the unit sphere $S^{n-1}=\left\{\eta \in \mathbb{R}_{\eta}^{n}:|\eta|=1\right\}$, with small enough support and with unit value around the point $\eta_{0} /\left|\eta_{0}\right|$. Then, by making the obvious homogeneous extension of degree 0 , for any $\eta$, cutting off to 0 around the origin to avoid smoothness problems, and finally multiplying by a bump function around $\left(x_{0}, y_{0}\right)$, one obtains a symbol $a \in S^{0}\left(\mathbb{R}_{x, y}^{2 n} \times \mathbb{R}_{\eta}^{n}\right)$ that satisfies all of the above requirements.

Based on the analysis made in these remarks, we now move towards setting up the appropriate conditions for the improved theorem. Actually, the only essential difference has to do with the Fourier integral 
operator used in the conjugation. Unlike the relative freedom of choice allowed in the classical version of Egorov's theorem, as observed in Remark iii), the higher accuracy now requires a very particular operator with unitary properties.

To begin with, we only need to be concerned with the canonical transformation of pseudodifferential operators at a microlocal level. As observed in Remark i), if one starts from a global symplectomorphism between cotangent bundles, a global version of the theorem can then be obtained from the microlocal one by using a locally finite partition of unity on the cosphere bundle of the product manifold. Therefore, following Remark iv), it will be enough to consider for the conjugation a local Fourier integral operator given by an oscillatory integral as in (19), but now with a more careful choice of symbol $a \in S^{0}\left(\mathbb{R}_{x, y}^{2 n} \times \mathbb{R}_{\eta}^{n}\right)$ in order to make it microlocally unitary.

Given $A \in I^{\mu}\left(X \times Y, C_{\Phi} ; \Omega_{X \times Y}^{1 / 2}\right)$, its adjoint $A^{*} \in I^{\mu}\left(Y \times X, C_{\Phi^{-1}} ; \Omega_{Y \times X}^{1 / 2}\right)$ is a Fourier integral operator of the same order as that of $A$, acting from half-densities on $X$ back to half-densities on $Y$, and associated to the graph of the inverse $\Phi^{-1}$. From the description in Remark ii) above, concerning the composition of Fourier integral operators associated to graphs of canonical transformations, if we want

$$
A^{*} A-I_{Y} \in \Psi^{-\infty}
$$

to be satisfied microlocally close to $\left(y_{0}, \eta_{0}\right) \in T^{*} Y \backslash 0$, then necessarily $\mu=0$ and the principal symbols should satisfy

$$
\sigma\left(A^{*}\right)_{(y, \eta, \Phi(y, \eta))} \sigma(A)_{\left.\right|_{(\Phi(y, \eta), y, \eta)}} \equiv 1 \quad\left(\bmod S^{-1}\right),
$$

in a conic neighborhood of $\left(y_{0}, \eta_{0}\right) \in T^{*} Y \backslash 0$. When $A$ is given by (19), the kernel of its adjoint is

$$
K_{A^{*}}(y, x)=\frac{1}{(2 \pi)^{n}} \int e^{i(y \cdot \eta-S(x, \eta))} \bar{a}(x, y, \eta) d \eta,
$$

and its principal symbol $\sigma\left(A^{*}\right) \in S^{0}\left(C_{\Phi^{-1}}, \mathcal{L}_{C_{\Phi^{-1}}}\right) / S^{-1}\left(C_{\Phi^{-1}}, \mathcal{L}_{C_{\Phi^{-1}}}\right)$ is

$$
\begin{aligned}
\sigma\left(A^{*}\right)_{(y, \eta, \Phi(y, \eta))=\left(\partial_{\eta} S(x, \eta), \eta, x, \partial_{x} S(x, \eta)\right)} & =\left.\overline{\sigma(A)}\right|_{(\Phi(y, \eta), y, \eta)} \\
& =\frac{\bar{a}\left(x, \partial_{\eta} S(x, \eta), \eta\right)}{\left|\operatorname{det} \frac{\partial^{2} S}{\partial x \partial \eta}(x, \eta)\right|^{1 / 2}},
\end{aligned}
$$


in the trivialization of the Keller-Maslov-Hörmander line bundle associated to the phase $y \cdot \eta-S(x, \eta)$ above. So $(21)$ reads

$$
|\sigma(A)|_{(\Phi(y, \eta), y, \eta)}^{2}-1=\frac{|a(x, y, \eta)|^{2}}{\left|\operatorname{det} \frac{\partial^{2} S}{\partial x \partial \eta}(x, \eta)\right|}-1 \in S^{-1},
$$

with $x$ now considered as a function of $(y, \eta)$, according to the canonical transformation $\Phi$, in order to regard this as a symbol for a pseudodifferential operator on $Y$. A natural choice for the symbol $a \in S^{0}\left(\mathbb{R}_{x, y}^{2 n} \times \mathbb{R}_{\eta}^{n}\right)$ is then to make it

$$
a(x, y, \eta)=\left|\operatorname{det} \frac{\partial^{2} S}{\partial x \partial \eta}(x, \eta)\right|^{1 / 2},
$$

in a conic neighborhood of $\left(x_{0}, \xi_{0}, y_{0}, \eta_{0}\right) \in C_{\Phi}$, thus making the invariant principal symbol $\sigma(A) \in S^{0}\left(C_{\Phi}, \mathcal{L}_{C_{\Phi}}\right) / S^{-1}\left(C_{\Phi}, \mathcal{L}_{C_{\Phi}}\right)$ equal to one, when $\mathcal{L}_{C_{\Phi}}$ is trivialized by $(18)$.

Technically, we still need to tailor this symbol a little bit, with an appropriate cut-off function. But it can be noticed, already, that this is precisely the same symbol as (8), in the example of the previous section, of the change of coordinates for pseudodifferential operators acting on half-densities.

Another instance of the appearance of this symbol, based on the generating function of the canonical transformation, comes from writing the metaplectic representation of the symplectic group, in integral form. The unitary invariance of the Weyl operator, under linear symplectic transformations of its symbol, can then be interpreted as an exact form of Egorov's theorem, while the metaplectic unitary operators used in the conjugation can be regarded as Fourier integral operators associated to linear symplectic transformations, with symbols again given by (22). This is precisely the motivation that we will recall and develop a little bit more in detail in the next section.

Actually, there is nothing very original about (22), which in the literature is a frequently found choice for the symbol, in the classical version of Egorov's theorem, if one wants to perform the conjugation of pseudodifferential operators by unitary (modulo $S^{m-1}$ ) Fourier integral operators. The novelty here is that we will show that this exact same symbol provides the higher accuracy (modulo $S^{m-2}$ ) of both the unitary property of $A$ as well as of Egorov's theorem. Moreover, from a coordinate invariant point of view the usage of (22) in the formula for the oscillatory integral (19) naturally makes this kernel a distribution halfdensity on $X \times Y$, just like we have already observed for the particular 
case of (6). Therefore, this again hints that the appropriate geometrical framework for using these unitary Fourier integral operators is to consider all the operators as acting on half-densities, like we do in the statement of our theorem.

For the final step of the construction of the particular Fourier integral operator to be used in the conjugation, one just needs to consider a suitable cut-off function $\chi$ in the $(x, y, \eta) \in \mathbb{R}_{x, y}^{2 n} \times \mathbb{R}_{\eta}^{n}$ variables, with value one in a small conic neighborhood of $\left(x_{0}, y_{0}, \eta_{0}\right)$, for large $\eta$, and value zero around $\eta=0$, to avoid singularities. The construction of such a cut-off function is exactly the same as the one presented at the end of Remark iv). So that, when multiplying (22) by it we get a smooth symbol of order 0 , elliptic at $\left(x_{0}, y_{0}, \eta_{0}\right) \in \mathbb{R}_{x, y}^{2 n} \times\left(\mathbb{R}_{\eta}^{n} \backslash 0\right)$, with support in a sufficiently small compactly generated cone around this point and totally contained in the $(x, y, \eta)$ domain of $(18)$. The relevant fact, that should be kept in mind when performing computations, is that in a small open conic neighborhood of $\left(x_{0}, y_{0}, \eta_{0}\right)$ and for large $\eta$ - which is really the region of concern - the symbol is exactly (22). The cutoff function only guarantees that everything is well defined, that the final symbol is smooth everywhere on $T^{*} Y$ and that its $(x, y)$ support is compact therefore ensuring the proper support of $A$.

In the same coordinate systems around $x_{0}$ and $y_{0}$ as in Remark iv), for which the existence of the generating function $S(x, \eta)$ that describes the canonical transformation is guaranteed, we then have the local form of the Schwartz kernel of the Fourier integral operator $A$ that we are going to use

(23) $K_{A}(x, y)=\frac{1}{(2 \pi)^{n}} \int e^{i(S(x, \eta)-y \cdot \eta)}\left|\operatorname{det} \frac{\partial^{2} S}{\partial x \partial \eta}(x, \eta)\right|^{1 / 2} \chi(x, y, \eta) d \eta$.

Obviously, we consider $K_{A}$ to be zero at the remaining points of $X \times Y$. Its wavefront set is contained only in a small conic neighborhood of $\left(x_{0}, \xi_{0}, y_{0}, \eta_{0}\right)$, on the graph $C_{\Phi}$.

At this point, based on the motivation presented in the following section of this article, we define the principal Weyl symbol of a pseudodifferential operator acting on half-densities as the more refined principal symbol for this type of operators, whose invariant definition on the cotangent bundle of the manifold was recalled in the last section. The canonical transformation of these symbols is precisely what represents the sharper statement of Egorov's theorem, to be proved ahead.

Definition 1. Let $P \in \Psi^{m}\left(X, \Omega_{X}^{1 / 2}\right)$ be a pseudodifferential operator of order $m \in \mathbb{R}$, acting on half-densities on the manifold $X$. Then, the 
symbol $\sigma^{w}(P) \in S^{m}\left(T^{*} X\right) / S^{m-2}\left(T^{*} X\right)$ which, for any local coordinate system on $X$, satisfies (according to the known invariance, mentioned in the previous section)

$$
\sigma^{w}(P)(x, \xi) \equiv p(x, \xi)+\frac{i}{2} \sum_{k=1}^{n} \partial_{x_{k}} \partial_{\xi_{k}} p(x, \xi) \quad\left(\bmod S^{m-2}\right),
$$

where $p(x, \xi) \in S^{m}$ is the complete symbol of $P$ in these coordinates, will be called the principal Weyl symbol of $P$.

We have now established the framework for stating the theorem as follows.

Theorem 1. Let $\Phi$ be a local homogeneous symplectomorphism as in (10) and $A \in I^{0}\left(X \times Y, C_{\Phi} ; \Omega_{X \times Y}^{1 / 2}\right)$ the Fourier integral operator associated to it constructed above, represented in appropriate coordinate systems around $y_{0} \in Y$ and $x_{0} \in X$ by the oscillatory integral (23), with its invariant principal symbol $\sigma(A) \in S^{0}\left(C_{\Phi}, \mathcal{L}_{C_{\Phi}}\right) / S^{-1}\left(C_{\Phi}, \mathcal{L}_{C_{\Phi}}\right)$ satisfying $\sigma(A)=1$ in a conic neighborhood of $\left(x_{0}, \xi_{0}, y_{0}, \eta_{0}\right) \in C_{\Phi}$, when $\mathcal{L}_{C_{\Phi}}$ is trivialized by the phase (18). Then,

$$
\begin{aligned}
& A^{*} A-I_{Y} \in \Psi^{m-2}, \\
& A A^{*}-I_{X} \in \Psi^{m-2},
\end{aligned}
$$

in a conic neighborhood of $\left(y_{0}, \eta_{0}\right) \in T^{*} Y \backslash 0$, for (25); in one of $\left(x_{0}, \xi_{0}\right)=$ $\Phi\left(y_{0}, \eta_{0}\right) \in T^{*} X \backslash 0$, for (26). And given any properly supported pseudodifferential operator $P \in \Psi^{m}\left(X, \Omega_{X}^{1 / 2}\right)$, of order $m \in \mathbb{R}$, acting on half-densities in $X$, the conjugation

$$
Q=A^{*} P A,
$$

yields a pseudodifferential operator $Q \in \Psi^{m}\left(Y, \Omega_{Y}^{1 / 2}\right)$ whose principal Weyl symbol is microlocally related to $P$ 's by

$$
\sigma^{w}(Q)=\sigma^{w}(P) \circ \Phi,
$$

in the same conic neighborhood of $\left(y_{0}, \eta_{0}\right)$ as (25). If $P$ is given by a classical symbol $p(x, \xi) \sim p_{m}(x, \xi)+p_{m-1}(x, \xi)+\cdots$, this shows that its subprincipal symbol $\operatorname{Sub}(P)(x, \xi)=p_{m-1}(x, \xi)+\frac{i}{2} \sum_{k=1}^{n} \partial_{x_{k}} \partial_{\xi_{k}} p_{m}(x, \xi)$ is also microlocally transformed under the local homogeneous symplectomorphism $\Phi$, besides the usual transformation of the principal symbol $p_{m}(x, \xi)$ which is already given by the classical version of Egorov's theorem.

We finish this section with one last observation. The unitary Fourier integral operator $A$ used in the conjugation, as constructed above, 
requires a certain freedom of choice of the local coordinates on $Y$, around $y_{0}$, in order to be written as (23) to ensure that the canonical transformation $\Phi$ can be described by a generating function $S(x, \eta)$. However, in some applications of Egorov's theorem one might have to work with specific canonical transformations in certain fixed coordinates, which might not be adequate for writing the generating function with variables $(x, \eta)$. In that case, it is not possible to define the unitary Fourier integral operator by the oscillatory integral (23) in those coordinates. Nevertheless, Theorem 1 is stated in coordinate invariant terms and it is only this explicit local formula for the operator $A$ that depends on suitable $y$ coordinates. So the existence itself of such $A$ is never in question, only its representation in the given fixed coordinates. Still, if one wants fully explicit formulas, then the only thing that has to be done is to consider a suitable change of coordinates on $Y$ just for that purpose. The procedure to be followed is exactly the one also used in the classical version of Egorov's theorem in the same circumstances, and that is to perform the conjugation in two steps: the first one is to apply Theorem 1 and do a conjugation of the original pseudodifferential operator on $X$ by a unitary operator defined by (23), considering new convenient coordinates on $Y$ for which the required generating function is guaranteed to exist; the second step is simply a change of coordinates on $Y$, conjugating this time by a unitary Fourier integral operator appropriate for that purpose, as in (6) (again a particular case of Theorem 1) in order to bring the canonically transformed pseudodifferential operator acting on half-densities on $Y$, produced in the previous step, from the new $y$ coordinates back to the original ones. Of course, the composition of the two unitary Fourier integral operators used in these two stages provides a single operator by which the initial pseudodifferential operator can be conjugated, to get at once to the final canonically transformed one.

\section{Motivation: Weyl correspondence, symplectic invariance and metaplectic representation}

The Weyl quantization is normally considered only for symbols globally defined on the whole of $\mathbb{R}^{n}$, thus providing an alternative symboloperator correspondence for pseudodifferential operators in $\mathbb{R}^{n}$. The Weyl operator associated to a symbol $a=a(x, \xi) \in \mathcal{S}\left(\mathbb{R}^{n} \times \mathbb{R}^{n}\right)$, for example, acting on a function $u \in \mathcal{S}\left(\mathbb{R}^{n}\right)$, is given by the formula

$$
a^{w}(x, D) u(x)=\frac{1}{(2 \pi)^{n}} \iint e^{i(x-y) \cdot \xi} a\left(\frac{x+y}{2}, \xi\right) u(y) d y d \xi
$$


whereas the standard pseudodifferential operator for the same symbol is given by

$$
\begin{aligned}
a(x, D) u(x) & =\frac{1}{(2 \pi)^{n}} \int e^{i x \cdot \xi} a(x, \xi) \hat{u}(\xi) d \xi \\
& =\frac{1}{(2 \pi)^{n}} \iint e^{i(x-y) \cdot \xi} a(x, \xi) u(y) d y d \xi
\end{aligned}
$$

By writing the kernels of these operators as oscillatory integrals

$$
\begin{aligned}
K_{a^{w}}(x, y) & =\frac{1}{(2 \pi)^{n}} \int e^{i(x-y) \cdot \xi} a\left(\frac{x+y}{2}, \xi\right) d \xi \\
& =\mathcal{F}_{2}^{-1}(a)\left(\frac{x+y}{2}, x-y\right),
\end{aligned}
$$

for $(27)$, and

$$
K_{a}(x, y)=\frac{1}{(2 \pi)^{n}} \int e^{i(x-y) \cdot \xi} a(x, \xi) d \xi=\mathcal{F}_{2}^{-1}(a)(x, x-y),
$$

for (28) (where $\mathcal{F}_{2}^{-1}$ means the inverse Fourier transform in the second variable only), these operators are naturally extended to symbols in $\mathcal{S}^{\prime}\left(\mathbb{R}^{n} \times \mathbb{R}^{n}\right)$. The two different maps, (29) and (30), from the symbols $a(x, \xi)$ to these two types of kernels, $K_{a}(x, y)$ and $K_{a^{w}}(x, y)$, are obviously isomorphisms in $\mathcal{S}^{\prime}\left(\mathbb{R}^{n} \times \mathbb{R}^{n}\right)$. Due to Schwartz's kernel theorem one then obtains two different bijections between these very general symbols in $\mathcal{S}^{\prime}\left(\mathbb{R}^{n} \times \mathbb{R}^{n}\right)$ and the continuous operators from $\mathcal{S}\left(\mathbb{R}^{n}\right)$ to $\mathcal{S}^{\prime}\left(\mathbb{R}^{n}\right)$ : they are called, respectively, the Weyl and the standard pseudodifferential operator correspondences (or quantizations). When restricted to $L^{2}\left(\mathbb{R}^{n} \times \mathbb{R}^{n}\right)$, these two correspondences are unitary isomorphisms between the set of symbols and the space of Hilbert-Schmidt operators on $L^{2}\left(\mathbb{R}^{n}\right)$.

Given a fixed continuous operator from $\mathcal{S}\left(\mathbb{R}^{n}\right)$ to $\mathcal{S}^{\prime}\left(\mathbb{R}^{n}\right)$, the unique symbol in $\mathcal{S}^{\prime}\left(\mathbb{R}^{n} \times \mathbb{R}^{n}\right)$ that yields it, according to the Weyl correspondence, is called the Weyl symbol of the operator. Analogously, one obtains the so called standard pseudodifferential symbol, for the same operator, by using the standard correspondence. 
Restricting from $\mathcal{S}^{\prime}\left(\mathbb{R}^{n} \times \mathbb{R}^{n}\right)$ to the usual classes of symbols of pseudodifferential calculus $a \in S^{m}\left(\mathbb{R}^{n} \times \mathbb{R}^{n}\right) \subset \mathcal{S}^{\prime}\left(\mathbb{R}^{n} \times \mathbb{R}^{n}\right), m \in \mathbb{R}$, which satisfy the global estimates (in the $x$ variable),

$$
\left|D_{x}^{\alpha} D_{\xi}^{\beta} a(x, \xi)\right| \leq C_{\alpha, \beta}(1+|\xi|)^{m-|\beta|},
$$

for all multi-indices $\alpha, \beta \in \mathbb{N}^{n}$ and $(x, \xi) \in \mathbb{R}^{n} \times \mathbb{R}^{n}$, the iterated integrals (27) and (28), or equivalently the oscillatory integral kernels (29) and (30), also define bijections between these symbol classes $S^{m}$ and a common set of pseudodifferential operators, continuous on $\mathcal{S}\left(\mathbb{R}^{n}\right)$ and extendable to $\mathcal{S}^{\prime}\left(\mathbb{R}^{n}\right)$. These are the global analogues to the local pseudodifferential operators, continuous on $C_{c}^{\infty}$ and extendable to $\mathcal{D}^{\prime}$, except that in the local theory, unlike here, there generally does not exist an exact bijection between symbols and operators (only modulo $S^{-\infty}$ ).

The conversion from one type of symbol to the other, for the same operator, is naturally called the rule for change of quantization. If $a, p \in$ $\mathcal{S}^{\prime}\left(\mathbb{R}^{n} \times \mathbb{R}^{n}\right)$ are such that $a^{w}(x, D)=p(x, D)$, as operators $\mathcal{S}\left(\mathbb{R}^{n}\right) \rightarrow$ $\mathcal{S}^{\prime}\left(\mathbb{R}^{n}\right)$, according to the two previous quantization procedures, then one merely has to equate the corresponding kernels to obtain

$$
K_{a^{w}}(x, y)=K_{p}(x, y) \Rightarrow \mathcal{F}_{2}^{-1}(a)\left(\frac{x+y}{2}, x-y\right)=\mathcal{F}_{2}^{-1}(p)(x, x-y),
$$

which yields the transformation rule, from the standard to the Weyl symbol,

$$
a(x, \xi)=e^{-\frac{i}{2} D_{x} \cdot D_{\xi}} p(x, \xi) .
$$

Here $e^{-\frac{i}{2} D_{x} \cdot D_{\xi}}$ represents the Fourier multiplier operator $\mathcal{S}^{\prime}\left(\mathbb{R}^{n} \times \mathbb{R}^{n}\right) \rightarrow$ $\mathcal{S}^{\prime}\left(\mathbb{R}^{n} \times \mathbb{R}^{n}\right)$ given by

$$
e^{-\frac{i}{2} D_{x} \cdot D_{\xi}} p(x, \xi)=\frac{1}{(2 \pi)^{2 n}} \int e^{i(\hat{x} \cdot x+\hat{\xi} \cdot \xi)} e^{-\frac{i}{2} \hat{x} \cdot \hat{\xi}} \hat{p}(\hat{x}, \hat{\xi}) d \hat{x} d \hat{\xi} .
$$

The inverse change of quantization, from the Weyl to the standard symbol, also follows immediately,

$$
p(x, \xi)=e^{\frac{i}{2} D_{x} \cdot D_{\xi}} a(x, \xi) .
$$

When restricted to $S^{m}$, the transformation (31), for change of quantization, is still very well behaved: it is a Fréchet space isomorphism. And, in this case, its image can actually be given by the convenient asymptotic expansion (see [Fol], [Hör3])

$$
a(x, \xi)=e^{-\frac{i}{2} D_{x} \cdot D_{\xi}} p(x, \xi) \sim \sum_{j=0}^{\infty} \frac{\left(i \partial_{x} \cdot \partial_{\xi}\right)^{j}}{2^{j} j !} p(x, \xi),
$$


which is what one would formally get by expanding the exponential in (31) in power series. Obviously, a totally analogous asymptotic expansion can also be obtained for the inverse transformation, from the Weyl to the standard symbol. In particular, at the leading order, both symbols are equal, i.e.

$$
a(x, \xi) \equiv p(x, \xi) \quad\left(\bmod S^{m-1}\left(\mathbb{R}^{n} \times \mathbb{R}^{n}\right)\right),
$$

whereas at the next order of accuracy (32) yields

$$
\begin{aligned}
a(x, \xi) & \equiv p(x, \xi)-\frac{i}{2} D_{x} \cdot D_{\xi} p(x, \xi) \\
& =p(x, \xi)+\frac{i}{2} \sum_{k=1}^{n} \partial_{x_{k}} \partial_{\xi_{k}} p(x, \xi) \quad\left(\bmod S^{m-2}\left(\mathbb{R}^{n} \times \mathbb{R}^{n}\right)\right) .
\end{aligned}
$$

Of course, if $p \in S^{m}\left(\mathbb{R}^{n} \times \mathbb{R}^{n}\right)$ is a classical symbol $p(x, \xi) \sim p_{m}(x, \xi)+$ $p_{m-1}(x, \xi)+\cdots$, with $p_{m-j}(x, \xi) \in S^{m-j}$ positively homogeneous of degree $m-j$ in the $\xi$ variable, for large $\xi$ and $j \in \mathbb{N}$, then $a \in S^{m}$ will also be a classical symbol, whose homogeneous terms of degree $m$ and $m-1$, in its asymptotic expansion, will respectively be the principal and subprincipal symbols of $p$,

$$
\begin{array}{rlrl}
a_{m}(x, \xi) & =p_{m}(x, \xi) & \\
a_{m-1}(x, \xi) & =p_{m-1}(x, \xi)+\frac{i}{2} \sum_{k=1}^{n} \partial_{x_{k}} \partial_{\xi_{k}} p_{m}(x, \xi) .
\end{array}
$$

The subprincipal symbol of an operator therefore appears naturally under the Weyl correspondence.

The reason for our choice of terminology, in Definition 1, is now clear. We call (24) the principal Weyl symbol of a pseudodifferential operator with complete (standard) symbol $p(x, \xi) \in S^{m}$ precisely because it is the same as (33), i.e., its Weyl symbol modulo $S^{m-2}$, obtained by the rule of change of quantization. Moreover, the properties of the Weyl calculus, that we will recall in the sequence, constitute the essential motivation for Theorem 1 and we will see that the role of (33) there can be interpreted as parallel to the role of the (standard) principal symbol in the classical version of Egorov's theorem. Including, as a particular case, the fact that they both are invariantly defined as functions on the cotangent bundle, within the right geometric framework.

In spite of the equivalence between the two types of symbol-operator correspondences discussed above, it is well known that the Weyl calculus presents several advantages over the standard calculus. In other words, the usage of the Weyl symbol for the pseudodifferential operators leads 
to well known benefits in the corresponding symbolic calculus ([DiSj], [Fol], [Hör1], [Hör3]). In a way, what we intend to do now is to provide some arguments that portray Theorem 1 as being yet another example of that fact.

The source of the advantages for the Weyl calculus lies in the symmetric approach taken in the integration of the symbol, where the average $(x+y) / 2$ is used in (27) instead of just $x$ in (28). This seemingly simple detail is deeply and crucially rooted in the underlying mathematical structure of the quantization problem, in connection with which Hermann Weyl first introduced the operator, in the late 1920's ([Wey]). The general goal of quantization is to establish a correspondence between the observables in classical mechanics - real functions defined on the symplectic phase space - and the observables in quantum mechanics - self-adjoint operators on the Hilbert phase space - that somehow best reflect the properties of their classical counterparts. There are several requirements, from the points of view both of mathematics and physics, that such a correspondence should fulfill and it is known that an ideal, sort of "isomorphic", type of association is impossible (see [Fol]). In fact, physically, according to Bohr's general principle, one should only expect to fully recover the classical description from the quantum system, in the semi-classical limit $\hbar \rightarrow 0$. Nevertheless, even away from this limit (at $\hbar=1$ ), the Weyl quantization is, in many aspects, a very satisfactory way of assigning operators to symbols, within this context. Because of this, (27) encodes a genetic suitability to the symplectic properties of phase space and is particularly well adjusted to being used in situations where those properties are relevant.

On the other hand, the standard pseudodifferential calculus had its origin in the mathematics of partial differential equations, and the integral formula (28) is simply a consequence of the common convention of writing the differential operators as a sum of derivatives on the right multiplied by coefficients on the left. Therefore, the underlying structure of phase space is not transposed into definition (28).

Intuitively, one should generally expect then the Weyl calculus to produce better results than the standard pseudodifferential calculus, whenever the intrinsic symplectic structure of phase space and its connection with the mathematical setting of quantum mechanics play a significant role in the situation being studied. 
From a mathematical-physics point of view, one can interpret Egorov's theorem as relating transformations of classical observables under automorphisms of phase space - symplectomorphisms - to the transformations of their quantized versions, under the corresponding automorphisms of the quantum system - conjugation by unitary operators. If the quantization were ideal, the quantum observables resulting from two symplecticaly equivalent symbols should then be unitarily equivalent, producing an exact form of Egorov's theorem, without any errors. But that is too much to hope for and in general, according to Bohr's principle, such an exact rule should only be true in the limit when $\hbar \rightarrow 0$. This is the point of view used when studying this theorem in semi-classical analysis (see [Rob]), where the goal mostly has to do with accurately estimating the difference between the conjugated and the canonically transformed operators, as a function of $\hbar$. In fact, the symplectic transformations there are normally considered to be classical Hamiltonian flows, whereas the unitary transformations are the unitary evolutions of the quantum system for the corresponding quantum Hamiltonian. And a great part of the study then (as in [BoRo]) concerns evaluating the accuracy of Egorov's theorem as a function, not only of $\hbar$, but also of time. Nevertheless, based on the advantages of the Weyl quantization in these circumstances, even without doing semi-classical limits and staying exclusively within the framework of classical microlocal analysis, one should still expect the Weyl correspondence to produce a better outcome in Egorov's theorem, than the standard pseudodifferential one, as used in the classical version of the theorem.

We now focus on two specific cases of the benefits that the difference in the integral formulas (27) and (28) subtly leads to, which are at the root of the mathematical reasoning that leads to expecting why the improvement of Egorov's theorem should hold, and in which circumstances. They are among the most important properties of the Weyl correspondence, that distinguish it from the standard pseudodifferential one.

We start by pointing out that the adjoint of a Weyl operator (with respect to the $L^{2}$ inner product) defined on $\mathcal{S}\left(\mathbb{R}^{n}\right)$, is another pseudodifferential operator whose Weyl symbol is simply the complex conjugate of the original one. This is in contrasting difference with the standard pseudodifferential operators, where the symbol of the adjoint is given generally by a much more complicated asymptotic expansion. As a direct result of this fact, the Weyl operator with symbols in $S^{m}\left(\mathbb{R}^{n} \times \mathbb{R}^{n}\right)$ is symmetric if and only if its symbol is real valued. (See [Fol] for extra conditions on the symbols which guarantee, actually, that the Weyl 
operator is essentially self-adjoint on $\mathcal{S}\left(\mathbb{R}^{n}\right)$.) Naturally, this is exactly the reason that makes (27) such a good model for the quantization of an observable in classical mechanics given by a real valued function in phase space.

The other fundamental property of the Weyl calculus, that we want to make a reference to, is its unitary invariance under linear symplectic transformations (or slightly more generally, under affine symplectic transformations). Let us suppose that we have $T \in S p(n, \mathbb{R})$, the symplectic group, and that we want to transform a Weyl operator by composing its symbol $a(x, \xi)$ with this linear symplectic transformation. Then, there exists an isomorphism $U_{T}$ on $\mathcal{S}^{\prime}\left(\mathbb{R}^{n}\right)$, whose restriction to $L^{2}\left(\mathbb{R}^{n}\right)$ is a unitary transformation, depending only on $T$ and unique up to multiplication by a constant factor of modulus 1 , such that the Weyl operator resulting from the composed symbol can simply be obtained conjugating the initial one by $U_{T}$,

$$
U_{T}^{-1} a^{w}(x, D) U_{T}=(a \circ T)^{w}(x, D) .
$$

A convenient choice of the multiplicative constant of modulo 1, for $U_{T}$ yields a homomorphism $T \mapsto U_{T}$, of the symplectic group into the unitary operators on $L^{2}\left(\mathbb{R}^{n}\right)$, which is called the metaplectic representation. For standard pseudodifferential operators, (34) would only hold true if the symbol $a(x, \xi)$ were a linear function of $(x, \xi) \in \mathbb{R}^{2 n}$ (a particular case for which both of these two symbol-operator correspondences coincide, anyway). On the other hand, this unitary equivalence for the symplectic group is universally true for the Weyl operator $a^{w}(x, D)$, using any symbol $a(x, \xi)$ defined on $\mathbb{R}^{2 n}$, including the very general case of tempered distributions. Clearly, this outstanding example of perfect behaviour with respect to symplectic transformations is one of those consequences of the fact that the mathematical framework of classical and quantum mechanics underlies the origin of the definition of the Weyl quantization. But what is more striking is that (34) really characterizes the Weyl operator. In other words, the Weyl correspondence is necessarily the only way to associate a pseudodifferential operator to a symbol, which fulfills the basic restrictions of mapping the coordinate functions $x$ and $\xi$, in phase space, to the operators $X$ (multiplication by $x)$ and $D(=-i \partial)$, and is unitarily invariant under linear symplectic transformations of that symbol, according to (34) (see [Fol], [Ste]).

Property (34) can obviously be interpreted as an exact form of Egorov's theorem, where the rule for the symbol transformation is achieved with absolutely no errors. And it shows that the usage of Weyl quantization instead of the standard pseudodifferential one, for 
the same operators, improves the accuracy of Egorov's theorem here, from just being true at the leading order of the symbols, to being fully exact.

A closer resemblance still, between (34) and Egorov's theorem in the setting of classical microlocal analysis, is obtained finally by writing the metaplectic representation of the symplectic group $U_{T}$ in integral form (in the Appendix to Chapter 7 , in $[\mathbf{D i S j}]$, the metaplectic operators are precisely used as a linear model introduction to the general theory of Fourier integral operators). Let $T \in S p(n, \mathbb{R})$ be a linear symplectic transformation in $\mathbb{R}^{n} \times \mathbb{R}^{n} \cong T^{*} \mathbb{R}^{n}$, say $(x, \xi)=T(y, \eta)$. Because the transformation is linear, the condition for the existence of a generating function in the variables $(x, \eta)$ is global and is equivalent to being able to parametrize the graph $C_{T}=\left\{(x, \xi, y, \eta) \in \mathbb{R}^{4 n}:(x, \xi)=T(y, \eta)\right\}$ of the transformation $T$ in these variables. And that happens if

$$
\operatorname{det}\left(\frac{\partial x}{\partial y}\right)=\operatorname{det}\left(\frac{\partial T_{x}}{\partial y}\right) \neq 0 \text {. }
$$

Supposing such a condition is satisfied, we can then write the generating function $S$, whose differential $d S=y \cdot d \eta+\xi \cdot d x$ is a closed 1-form on $C_{T}$, as a function of $(x, \eta)$ for the whole $\mathbb{R}^{2 n}$. And we have, on the points of the graph

$$
y_{j}=\frac{\partial S}{\partial \eta_{j}} \quad \text { and } \quad \xi_{j}=\frac{\partial S}{\partial x_{j}}, \quad j=1, \ldots, n .
$$

Notice how $S(x, \eta)$, in this particular case of linear symplectic transformations, is just a quadratic polynomial in $x$ and $\eta$. The condition (35) can be phrased, in terms of the generating function, by the fact that the (constant) matrix

$$
C=\frac{\partial^{2} S}{\partial x \partial \eta}
$$

is nonsingular.

If we now define an operator $U_{T}$ on $\mathcal{S}\left(\mathbb{R}^{n}\right)$ as

$$
U_{T} f(x)=\frac{1}{(2 \pi)^{n}} \int e^{i S(x, \eta)}|\operatorname{det} C|^{1 / 2} \hat{f}(\eta) d \eta, \quad f \in \mathcal{S}\left(\mathbb{R}^{n}\right),
$$

we get an object very similar to a Fourier integral operator of order zero except that, unlike in the definitions of the classical microlocal analysis, the generating function $S(x, \eta)$ is quadratic in $\eta$ and therefore does not necessarily produce a homogeneous phase of degree one. It can easily be shown that $U_{T}$ preserves the $L^{2}$ norm of $f$, i.e., $\left\|U_{T} f\right\|_{L^{2}\left(\mathbb{R}^{n}\right)}=\|f\|_{L^{2}\left(\mathbb{R}^{n}\right)}$ and so can be extended as a unitary operator on $L^{2}\left(\mathbb{R}^{n}\right)$. With a little 
further work, it can also be shown that (see [Fol, Chapter 4], also for another derivation of (36))

$$
U_{T}^{-1} a^{w}(x, D) U_{T}=(a \circ T)^{w}(x, D),
$$

i.e., that (36) represents the metaplectic operator associated to the linear symplectic transformation $T$. So we have written the unitary invariance of the Weyl operator (34) under the symplectic group $S p(n, \mathbb{R})$ in a form which is very similar to Egorov's theorem in the framework of microlocal analysis, when the linear canonical transformations have a corresponding generating function. The unitary operator $U_{T}$ is essentially a Fourier integral operator of order zero associated to the canonical transformation, with symbol equal to $\left|\operatorname{det} \partial^{2} S / \partial x \partial \eta\right|^{1 / 2}$, essentially the same as in Theorem 1.

Putting all these pieces together, the heuristic argument for the validity of Theorem 1 now follows from these two properties. On the one hand, real Weyl symbols produce self-adjoint operators, and conjugation by unitary operators preserves that self-adjointness. Therefore, if the starting Weyl symbol is real, then the Weyl symbol of the conjugated operator should still be real valued too. On the other hand, from basic pseudodifferential symbol calculus, it is well known that the asymptotic expansions that relate the final symbol to the original one produce a second order term that comes multiplied by $i$ which, in the case of real symbols, should therefore vanish. Intuitively, then, the Weyl symbol for the conjugated operator should generally equal the original one composed with the symplectomorphism, with a vanishing second order term. I.e., by using the Weyl correspondence instead of the standard pseudodifferential one, Egorov's theorem should be improved to an accuracy $S^{m-2}$ instead of the classical $S^{m-1}$. And this can be interpreted as an advantage of the Weyl quantization similar to the one obtained in (34), except that we are now considering general canonical transformations besides linear ones.

Finally, for Theorem 1, it is now simply a matter of translating these properties of the Weyl calculus in $\mathbb{R}^{n}$, to the setting of pseudodifferential operators on manifolds. The global integral formula (27) can no longer be used for the locally defined operators, so we keep the symbol correspondence (32) and drop the integral representation (27). Moreover, because we are only expecting a general accuracy improvement down to $S^{m-2}$, we really only need symbols with the same level of accuracy. That is why we end up defining the principal Weyl symbol that way, in Definition 1, disregarding lower orders. Because our heuristic argument is strongly based on the self-adjointness of the pseudodifferential 
operators with real Weyl symbols, the final geometric framework needs to reproduce this fact, which is done in coordinate invariant terms (on general manifolds without a distinguished positive density) by considering pseudodifferential operators acting on half-densities. That way, their adjoint operator is also of the same type (unlike for operators acting on functions, whose adjoints act on densities) and their principal Weyl symbols, well known to be invariantly defined on the cotangent bundle of the manifold, as explained in Section 1.1, are the complex conjugate of the original ones (see Theorems 18.1.33 and 18.1.34 in [Hör3]) exactly as it happens for the Weyl calculus in $\mathbb{R}^{n}$.

We have thus been able to keep the essential features of the Weyl quantization, that yield Theorem 1, in a full framework of classical microlocal analysis.

\section{Proof of Theorem 1}

This section is exclusively devoted to the proof of Theorem 1 .

Proof: We will work in those fixed coordinate systems, around $y_{0}$ and $x_{0}$, where the Fourier integral operator $A$ is written locally as (23), with the aid of the generating function of $C_{\Phi}$. Consequently, in the same coordinates, $A^{*}$ is given by the kernel ${ }^{3}$

$$
K_{A^{*}}(y, x)=\frac{1}{(2 \pi)^{n}} \int e^{i(y \cdot \eta-S(x, \eta))}\left|\operatorname{det} \frac{\partial^{2} S}{\partial x \partial \eta}(x, \eta)\right|^{1 / 2} \chi(x, y, \eta) d \eta
$$

We denote by

$$
p(x, \xi) \in S^{m}\left(\tilde{X} \times \mathbb{R}^{n}\right) / S^{-\infty}\left(\tilde{X} \times \mathbb{R}^{n}\right)
$$

the complete symbol of $P \in \Psi^{m}\left(X, \Omega_{X}^{1 / 2}\right)$, in those coordinates around $x_{0} . \tilde{X}$ here is the open set in $\mathbb{R}^{n}$ diffeomorphic to the coordinate neighborhood on $X$. Modulo a smoothing operator, $P$ can be represented locally by the oscillatory integral, with a properly supported kernel,

$$
\begin{aligned}
P u(x) & =\frac{1}{(2 \pi)^{n}} \iint e^{i(x-\tilde{x}) \cdot \xi} p(x, \xi) u(\tilde{x}) d \tilde{x} d \xi \\
& =\frac{1}{(2 \pi)^{n}} \int e^{i x \cdot \xi} p(x, \xi) \hat{u}(\xi) d \xi,
\end{aligned}
$$

\footnotetext{
${ }^{3}$ To simplify the notation, and because the coordinate systems around $x_{0}$ and $y_{0}$ are fixed in the whole proof, we will use the same letters to denote both the points on the manifolds $X$ and $Y$ as well as their coordinates in $\mathbb{R}^{n}$.
} 
for $u \in C_{c}^{\infty}\left(X, \Omega_{X}^{1 / 2}\right)$, with support contained in the coordinate neighborhood of $x_{0}$.

We know, from the theorem on composition of Fourier integral operators associated to graphs of canonical transformations, that $P A$ yields a Fourier integral operator $B \in I^{m}\left(X \times Y, C_{\Phi} ; \Omega_{X \times Y}^{1 / 2}\right)$, of order $m$, associated to $C_{\Phi}$. The asymptotic expansion formulas for this particular type of composition, of a pseudodifferential operator and a Fourier integral operator, are widely available in the literature $([\mathbf{G r S j}],[\mathbf{H o ̈ r} \mathbf{4}],[\mathbf{S h u}]$, [Tre]). They are usually derived from the application of the stationary phase method, as in Theorem 7.7.7 of [Hör2]. From these, we immediately obtain the result that $B=P A$ can be locally represented by an oscillatory integral, with the same phase as $A$,

$$
P A u(x)=B u(x)=\frac{1}{(2 \pi)^{n}} \iint e^{i(S(x, \eta)-\tilde{y} \cdot \eta)} b(x, \tilde{y}, \eta) u(\tilde{y}) d \tilde{y} d \eta
$$

for $u \in C_{c}^{\infty}\left(Y, \Omega_{Y}^{1 / 2}\right)$, with support contained in the coordinate neighborhood around $y_{0}$. The symbol $b \in S^{m}$ is given by an asymptotic expansion which, to order $m-2$, is written as

$$
\begin{gathered}
b(x, \tilde{y}, \eta) \equiv p\left(x, S_{x}^{\prime}(x, \eta)\right) a(x, \tilde{y}, \eta)-i \sum_{k=1}^{n} \partial_{\xi_{k}} p\left(x, S_{x}^{\prime}(x, \eta)\right) \partial_{x_{k}} a(x, \tilde{y}, \eta) \\
-\frac{i}{2} \sum_{j, k=1}^{n} \frac{\partial^{2} p}{\partial \xi_{j} \partial \xi_{k}}\left(x, S_{x}^{\prime}(x, \eta)\right) \frac{\partial^{2} S}{\partial x_{j} \partial x_{k}}(x, \eta) a(x, \tilde{y}, \eta) \quad\left(\bmod S^{m-2}\right),
\end{gathered}
$$

where

$$
a(x, y, \eta)=\left|\operatorname{det} \frac{\partial^{2} S}{\partial x \partial \eta}(x, \eta)\right|^{1 / 2} \chi(x, y, \eta) \in S^{0}
$$

from $(23)$

The conjugation $A^{*} P A=A^{*} B$ then produces a Fourier integral operator $Q$, of the same order as $P$, associated to the canonical transformation $\Phi^{-1} \circ \Phi=I_{T^{*} Y}$. So that $Q \in \Psi^{m}\left(Y, \Omega_{Y}^{1 / 2}\right)$. In local coordinates we have

$$
\begin{aligned}
Q u(y) & =A^{*} P A u(y)=A^{*} B u(y) \\
& =\frac{1}{(2 \pi)^{n}} \iint e^{i(y-\tilde{y}) \cdot \eta} c(y, \tilde{y}, \eta) u(\tilde{y}) d \tilde{y} d \eta,
\end{aligned}
$$


where $c(y, \tilde{y}, \eta)$ is given by the oscillatory integral in the $x, \zeta$ variables

(40) $c(y, \tilde{y}, \eta)=\frac{1}{(2 \pi)^{n}} \iint e^{i[y \cdot(\zeta-\eta)-S(x, \zeta)+S(x, \eta)]} a(x, y, \zeta) b(x, \tilde{y}, \eta) d x d \zeta$,

with $a(x, y, \eta) \in S^{0}$ still given by formula (39), now as a consequence of (37), and $b(x, y, \eta) \in S^{m}$ given by (38). The integration in the $x$ variable is a perfectly normal one because due to the construction of the symbol $a$, in particular of the cut-off function $\chi$, the $x$ support of the integrand in (40) is compact and contained in the corresponding coordinate neighborhood at $x_{0}$. The integration in the $\zeta$ variable, on the other hand, has to be regarded as an oscillatory integral. As such, it should be taken as a limit of a sequence of similar integrals with symbols $a_{j}$ in $S^{-\infty}$, with $a_{j} \rightarrow a$ in $S^{0+}$, that can simply be done by using, for example, a cut-off function of increasing support in $\zeta$. This is an absolutely standard procedure, so we will not dwell further on these technical details here.

The crucial step of this proof is obviously the computation of the asymptotic expansion for this symbol $c(y, \tilde{y}, \eta)$, in terms of the symbols $a, b$ and the generating function $S$. For the classical version of Egorov's theorem only the leading order term would be needed, which is quite straightforward to determine. But the accuracy of our result requires explicit formulas for the lower order terms in the expansion, whose computation demands a lot more effort. We provide this result in the following lemma.

Lemma 2. Let $a \in S^{0}$ and $b \in S^{m}$ as above. Then, the oscillatory integral (40) defines a symbol $c \in S^{m}$ which, modulo symbols in $S^{m-2}$, can be written as

$$
\begin{aligned}
c(y, \tilde{y}, \eta) \equiv & \frac{1}{\left|\operatorname{det} \frac{\partial^{2} S}{\partial x \partial \eta}(x, \eta)\right|} \\
& \times\left(C_{0,0}(y, \tilde{y}, \eta)+\frac{i}{2}\left[C_{1,0}(y, \tilde{y}, \eta)\right.\right. \\
& \left.\left.\quad+C_{1,1}(y, \tilde{y}, \eta)+C_{1,2}(y, \tilde{y}, \eta)\right]\right) \quad\left(\bmod S^{m-2}\right),
\end{aligned}
$$


where $C_{0,0} \in S^{m}$ and $C_{1,0}, C_{1,1}, C_{1,2} \in S^{m-1}$ are given by

$C_{0,0}(y, \tilde{y}, \eta)=a(x, y, \eta) b(x, \tilde{y}, \eta)$,

$$
\begin{aligned}
C_{1,0}(y, \tilde{y}, \eta)= & \sum_{i, j, k, l=1}^{n} V_{i j} U_{i k}^{-1} U_{j l}^{-1} \partial_{x_{k}} \partial_{x_{l}}(a(x, y, \eta) b(x, \tilde{y}, \eta)) \\
& -2 \sum_{i, j=1}^{n} U_{i j}^{-1} \partial_{x_{j}}\left(\partial_{\eta_{i}} a(x, y, \eta) b(x, \tilde{y}, \eta)\right),
\end{aligned}
$$

$C_{1,1}(y, \tilde{y}, \eta)=\sum_{i, j, k, l=1}^{n} U_{i j}^{-1} U_{k l}^{-1}\left(2 \frac{\partial^{3} S}{\partial x_{j} \partial x_{l} \partial \eta_{i}}\left(\partial_{\eta_{k}} a\right) b\right.$

$$
\begin{array}{r}
\left.+2 \frac{\partial^{3} S}{\partial x_{j} \partial \eta_{i} \partial \eta_{k}} \partial_{x_{l}}(a b)+\frac{\partial^{4} S}{\partial x_{j} \partial x_{l} \partial \eta_{i} \partial \eta_{k}} a b\right) \\
-\sum_{i, j, k, l, p, q=1}^{n} V_{i j} U_{i k}^{-1} U_{j l}^{-1} U_{p q}^{-1}\left(2 \frac{\partial^{3} S}{\partial x_{k} \partial x_{q} \partial \eta_{p}} \partial_{x_{l}}(a b)\right. \\
\left.+\frac{\partial^{3} S}{\partial x_{k} \partial x_{l} \partial \eta_{p}} \partial_{x_{q}}(a b)+\frac{\partial^{4} S}{\partial x_{k} \partial x_{l} \partial x_{q} \partial \eta_{p}} a b\right),
\end{array}
$$

$C_{1,2}(y, \tilde{y}, \eta)=\sum_{i, j, k, l, p, q, r, s=1}^{n} V_{i j} U_{i k}^{-1} U_{j l}^{-1} U_{p q}^{-1} U_{r s}^{-1}\left(\frac{\partial^{3} S}{\partial x_{k} \partial x_{q} \partial \eta_{p}} \frac{\partial^{3} S}{\partial x_{l} \partial x_{s} \partial \eta_{r}}\right.$

$$
\begin{aligned}
& +\frac{\partial^{3} S}{\partial x_{k} \partial x_{q} \partial \eta_{r}} \frac{\partial^{3} S}{\partial x_{l} \partial x_{s} \partial \eta_{p}} \\
& \left.+\frac{\partial^{3} S}{\partial x_{k} \partial x_{l} \partial \eta_{p}} \frac{\partial^{3} S}{\partial x_{q} \partial x_{s} \partial \eta_{r}}\right) a b
\end{aligned}
$$$$
-\sum_{i, j, k, l, p, q=1}^{n} U_{i j}^{-1} U_{k l}^{-1} U_{p q}^{-1}\left(\frac{\partial^{3} S}{\partial x_{l} \partial x_{q} \partial \eta_{i}} \frac{\partial^{3} S}{\partial x_{j} \partial \eta_{p} \partial \eta_{k}}\right.
$$$$
\left.+2 \frac{\partial^{3} S}{\partial x_{j} \partial x_{q} \partial \eta_{i}} \frac{\partial^{3} S}{\partial x_{l} \partial \eta_{k} \partial \eta_{p}}\right) a b
$$

$U$ and $V$ denote the matrices $\left(\frac{\partial^{2} S}{\partial x_{i} \partial \eta_{j}}(x, \eta)\right)_{1 \leq i, j \leq n}$ and $\left(\frac{\partial^{2} S}{\partial \eta_{i} \partial \eta_{j}}(x, \eta)\right)_{1 \leq i, j \leq n}$, respectively, and every occurrence of $x$, in the 
formulas above, is to be evaluated as the $x=x(y, \eta)$ component of the canonical transformation $(x, \xi)=\Phi(y, \eta)$, implicitly given by the equation $y=\partial_{\eta} S(x, \eta)$.

Proof: We will follow the usual approach for computing asymptotic expansions of symbols defined by oscillatory integrals, based on the method of stationary phase. Because we are interested in obtaining explicit formulas beyond the first term of the expansion, we will not pursue the more common technique of applying the Morse lemma to transform the phase into a quadratic form, for it deals with implicitly defined coordinate transformations that are not convenient for our lower order computations. Instead, we will use Theorems 7.7.5 and 7.7.6 in [Hör2], where an alternative proof is provided with which asymptotic expansions of a totally explicit nature can be obtained.

We will consider $|\eta| \geq 1$. Rewriting (40), by doing a change of variables $\theta=(\zeta-\eta) /|\eta|$ and $\tilde{\eta}=\eta /|\eta|$, one gets

(42) $\quad c(y, \tilde{y}, \eta)$

$$
=\frac{|\eta|^{n}}{(2 \pi)^{n}} \iint e^{i|\eta|[y \cdot \theta-S(x, \tilde{\eta}+\theta)+S(x, \tilde{\eta})]} a(x, y,|\eta|(\theta+\tilde{\eta})) b(x, \tilde{y},|\eta| \tilde{\eta}) d x d \theta,
$$

where we have used the homogeneity of the generating function $S$, with respect to the second variable, in the exponential.

The phase

$$
f(x, \theta ; y, \tilde{\eta})=y \cdot \theta-S(x, \tilde{\eta}+\theta)+S(x, \tilde{\eta})
$$

has critical points $(x, \theta)$ given, as functions of the parameters $(y, \tilde{\eta})$, by

$$
\left\{\begin{array}{l}
f_{x}^{\prime}=0 \Rightarrow-S_{x}^{\prime}(x, \tilde{\eta}+\theta)+S_{x}^{\prime}(x, \tilde{\eta})=0, \\
f_{\theta}^{\prime}=0 \Rightarrow y-S_{\eta}^{\prime}(x, \tilde{\eta}+\theta)=0 .
\end{array}\right.
$$

And using the properties of the generating function, these two equations in turn yield

$$
\left\{\begin{array}{l}
\theta=0, \\
y=S_{\eta}^{\prime}(x, \tilde{\eta})=S_{\eta}^{\prime}(x, \eta) \Rightarrow x=x(y, \tilde{\eta})=x(y, \eta),
\end{array}\right.
$$

where we write $x(y, \eta)$ to denote the $x$ component of the canonical transformation $\Phi$.

The Hessian matrix of the phase, at these points, is now given by

$$
f_{x, \theta}^{\prime \prime}=\left[\begin{array}{cc}
0 & -S_{x, \eta}^{\prime \prime}(x, \tilde{\eta}) \\
-S_{\eta, x}^{\prime \prime}(x, \tilde{\eta}) & -S_{\eta}^{\prime \prime}(x, \tilde{\eta})
\end{array}\right]=\left[\begin{array}{cc}
0 & -U \\
-{ }^{t} U & -V
\end{array}\right]
$$


where we have used for the blocks the same notation as in the statement of the lemma,

$$
\begin{gathered}
U=S_{x, \eta}^{\prime \prime}(x, \tilde{\eta})=\left(\frac{\partial^{2} S}{\partial x_{i} \partial \eta_{j}}(x, \tilde{\eta})\right)_{1 \leq i, j \leq n}, \\
{ }^{t} U=S_{\eta, x}^{\prime \prime}(x, \tilde{\eta})=\left(\frac{\partial^{2} S}{\partial \eta_{i} \partial x_{j}}(x, \tilde{\eta})\right)_{1 \leq i, j \leq n}, \\
V=S_{\eta}^{\prime \prime}(x, \tilde{\eta})=\left(\frac{\partial^{2} S}{\partial \eta_{i} \partial \eta_{j}}(x, \tilde{\eta})\right)_{1 \leq i, j \leq n},
\end{gathered}
$$

and the point $x$ is to be evaluated at $x=x(y, \tilde{\eta})=x(y, \eta)$. The determinant of (44) is therefore

$$
\operatorname{det} f_{x, \theta}^{\prime \prime}=\left(\operatorname{det} \frac{\partial^{2} S}{\partial x \partial \eta}\right)^{2} \neq 0,
$$

proving that the critical points are non-degenerate.

Before we can finally use the asymptotic expansion formulas, resulting from the application of the method of stationary phase, to estimate (42), we still need to make the integrand compactly supported in the $\theta$ variable (the support in $x$ is already compact, from the start). For that, one follows the usual approach of splitting up the integral into two terms, with a cut-off function $\alpha(\theta)$ which is one, for $|\theta| \leq 1$, and zero, for $|\theta| \geq 2$, for example. The part of the integral with the $(1-\alpha)$ term is then equal to zero in a neighborhood of the critical points (43), so that integrating by parts in the $x$ variable one easily shows that this term is rapidly decreasing in $|\eta|$. We are left, therefore, with the other term where the original integrand is multiplied by $\alpha$ and in whose support lie the critical points, thus leading to the relevant part of the asymptotic expansion. In a neighborhood of these points we have $\alpha=1$, so for all practical purposes it is as if the integral remained exactly the same as in (42). Because of that, and to simplify the notation, we will not write the cut-off function $\alpha$ explicitly in the formulas, just keeping in mind that the integrand is now compactly supported in both of the integrated variables, $x$ and $\theta$.

The conditions are now set to apply Theorems 7.7.5 and 7.7.6 in [Hör2], to get the explicit asymptotic expansion of (42) down to order $m-2$. Of course, an important part of the proof has to do with showing that $c$ is actually a symbol in $S^{m}$, given by such an expansion in the sense of symbolic calculus. But that follows from a standard argument, by using the estimates of the remainder terms in these 
stationary phase formulas, and applying the usual result in the symbolic calculus (like Theorem 1.9 in [GrSj], Theorem 18.1.4 in [Hör3], or Proposition 3.6 in $[\mathbf{S h u}])$ that allow one to prove asymptotic expansions of symbols without actually having to get estimates for all derivatives (see $[\mathbf{G r S j}]$ for several instances of the application of this argument).

So we will just concentrate on computing the expansion itself. We then get, from the application of these theorems,

$$
\begin{aligned}
c(y, \tilde{y}, \eta) \equiv & \frac{e^{i \frac{\pi}{4} \operatorname{sgn} f_{x, \theta}^{\prime \prime}(x(y, \tilde{\eta}), 0)}}{\left|\operatorname{det} f_{x, \theta}^{\prime \prime}(x(y, \tilde{\eta}), 0)\right|^{1 / 2}} \\
& \times \sum_{j=0}^{1} \sum_{0 \leq k \leq 2 j} \frac{(i|\eta|)^{-j}}{2^{j+k} k !(j+k) !} \\
& \times\left(g^{k} a b\right)_{\mid(x(y, \tilde{\eta}), 0)} \quad\left(\bmod S^{m-2}\right) .
\end{aligned}
$$

The auxiliary function $g(x, \theta ; y, \tilde{\eta})$ is the remainder term in the Taylor formula of order two for the phase, centered at the critical points:

$$
\begin{aligned}
g(x, \theta ; y, \tilde{\eta})= & f(x, \theta ; y, \tilde{\eta})-f(x(y, \tilde{\eta}), 0 ; y, \tilde{\eta}) \\
& -\frac{1}{2} f_{x, \theta}^{\prime \prime}(x(y, \tilde{\eta}), 0)(x-x(y, \tilde{\eta}), \theta) \cdot(x-x(y, \tilde{\eta}), \theta),
\end{aligned}
$$

so it vanishes of third order when $\theta=0$ and $x=x(y, \tilde{\eta})$.

The signature of the Hessian (44) is 0 . This can be deduced from the fact that the continuous family of non-singular block matrices

$$
\left[\begin{array}{cc}
0 & -U \\
-{ }^{t} U & -\tau V
\end{array}\right],
$$

for $\tau \in[0,1]$, has an invariant signature. Which can easily be seen to equal zero, in the simpler case of $\tau=0$.

We can now start computing the terms in (46). The grouping of the terms in (41), as well as the notation used, naturally correspond to the possible pairs $j, k$ in (46).

The first term is in $S^{m}$ and corresponds to $j=0$ for which $k$ can only be zero too. This yields

$$
\begin{array}{r}
\frac{1}{\left|\operatorname{det} f_{x, \theta}^{\prime \prime}(x(y, \tilde{\eta}), 0)\right|^{1 / 2}} a(x(y, \tilde{\eta}), y,|\eta| \tilde{\eta}) b(x(y, \tilde{\eta}), \tilde{y},|\eta| \tilde{\eta}) \\
=\frac{a(x(y, \eta), y, \eta) b(x(y, \eta), \tilde{y}, \eta)}{\left|\operatorname{det} \frac{\partial^{2} S}{\partial x \partial \eta}(x(y, \eta), \eta)\right|},
\end{array}
$$

from which we get the formula for $C_{0,0}(y, \tilde{y}, \eta)$. 
The next terms in the expansion, with symbols in $S^{m-1}$, correspond to the cases $j=1$ and $k=0,1,2$ in (46). These require the explicit computation of $\left(f_{x, \theta}^{\prime \prime}\right)^{-1}$ which can be determined from (44):

$$
f_{x, \theta}^{\prime \prime}(x(y, \tilde{\eta}), 0)^{-1}=\left[\begin{array}{cc}
{ }^{t} U^{-1} V U^{-1} & -{ }^{t} U^{-1} \\
-U^{-1} & 0
\end{array}\right] .
$$

We now handle each of these cases in turn.

$j=1, k=0$ :

For this case we get

$$
\begin{aligned}
& \frac{1}{2 i|\eta|\left|\operatorname{det} \frac{\partial^{2} S}{\partial x \partial \eta}\right|}\left({ }^{t} U^{-1} V U^{-1} D_{x} \cdot D_{x}-2 U^{-1} D_{x} \cdot D_{\theta}\right)(a b)_{\mid(x(y, \tilde{\eta}), 0)} \\
= & \frac{-i}{2|\eta|\left|\operatorname{det} \frac{\partial^{2} S}{\partial x \partial \eta}\right|}\left(-V U^{-1} \partial_{x} \cdot U^{-1} \partial_{x}+2 U^{-1} \partial_{x} \cdot \partial_{\theta}\right)(a b)_{\mid(x(y, \tilde{\eta}), 0)} .
\end{aligned}
$$

The $|\eta|$ term, in the denominator, gets included in $V$ due to the homogeneity of degree -1 of the entries of this matrix, and gets canceled by a multiplying factor of $|\eta|$ that appears as a result of the $\partial_{\theta}$ derivative operating on $a(x, y,|\eta|(\theta+\tilde{\eta}))$. So that all the functions can be finally evaluated at $x=x(y, \eta)$ and $\eta$, and we get $C_{1,0}(y, \tilde{y}, \eta)$ by writing the above formulas in component notation.

$j=1, k=1:$

Here (46) gives

$$
\begin{aligned}
& \frac{1}{8 i|\eta|\left|\operatorname{det} \frac{\partial^{2} S}{\partial x \partial \eta}\right|}\left({ }^{t} U^{-1} V U^{-1} D_{x} \cdot D_{x}-2 U^{-1} D_{x} \cdot D_{\theta}\right)^{2}(g a b)_{\mid(x(y, \tilde{\eta}), 0)} \\
= & \frac{-i}{|\eta|\left|\operatorname{det} \frac{\partial^{2} S}{\partial x \partial \eta}\right|}\left(\frac{1}{8}\left(V U^{-1} \partial_{x} \cdot U^{-1} \partial_{x}\right)^{2}-\frac{1}{2}\left(V U^{-1} \partial_{x} \cdot U^{-1} \partial_{x}\right)\left(U^{-1} \partial_{x} \cdot \partial_{\theta}\right)\right. \\
& \left.+\frac{1}{2}\left(U^{-1} \partial_{x} \cdot \partial_{\theta}\right)^{2}\right)(g a b)_{\mid(x(y, \tilde{\eta}), 0)} .
\end{aligned}
$$

Because $g$ has a third order zero at the critical points, for the fourth order differential operators not to annihilate the term $g a b$, at least three derivatives have to hit $g$. But then, from (47), it is clear that to compute those derivatives, we can simply substitute $g$ for $-S(x, \tilde{\eta}+\theta)+S(x, \tilde{\eta})$. However, if only derivatives in $x$ were involved, at the critical point $\theta=0$ 
these two terms would cancel each other. So we are reduced to the case where at least three derivatives hit $g$, necessarily in mixed combinations of $x$ and $\theta$. And these can be computed by just considering the function $-S(x, \tilde{\eta}+\theta)$.

So the term that only includes derivatives in $x$ vanishes and brings no contribution to the final formula. For the remaining two differential operators, after grouping together symmetric contributions of derivatives, we obtain the formula for $C_{1,1}(y, \tilde{y} \cdot \eta)$.

$j=1, k=2$ :

Finally, for this last case we get

$$
\begin{aligned}
\frac{1}{96 i|\eta|\left|\operatorname{det} \frac{\partial^{2} S}{\partial x \partial \eta}\right|}\left({ }^{t} U^{-1} V U^{-1} D_{x} \cdot D_{x}\right. & \left.-2 U^{-1} D_{x} \cdot D_{\theta}\right)^{3}\left(g^{2} a b\right)_{\mid(x(y, \tilde{\eta}), 0)} \\
=\frac{i}{|\eta|\left|\operatorname{det} \frac{\partial^{2} S}{\partial x \partial \eta}\right|}\left(\frac{1}{96}\left(V U^{-1} \partial_{x} \cdot U^{-1} \partial_{x}\right)^{3}\right. & -\frac{1}{16}\left(V U^{-1} \partial_{x} \cdot U^{-1} \partial_{x}\right)^{2}\left(U^{-1} \partial_{x} \cdot \partial_{\theta}\right) \\
& +\frac{1}{8}\left(V U^{-1} \partial_{x} \cdot U^{-1} \partial_{x}\right)\left(U^{-1} \partial_{x} \cdot \partial_{\theta}\right)^{2} \\
& \left.-\frac{1}{12}\left(U^{-1} \partial_{x} \cdot \partial_{\theta}\right)^{3}\right)\left(g^{2} a b\right)_{\mid(x(y, \tilde{\eta}), 0)} .
\end{aligned}
$$

The function $g^{2}$ has a sixth order zero at the critical points, so all the derivatives have to hit it, with the product $a b$ not being differentiated at all. Again, gathering together symmetric contributions of derivatives, and disregarding the first two sixth order differential operators here, that would bring only derivatives in $x$ to at least one of the factors of $g$, we obtain $C_{1,2}(y, \tilde{y}, \eta)$.

As $Q=A^{*} P A \in \Psi^{m}\left(Y, \Omega_{Y}^{1 / 2}\right)$ is a pseudodifferential operator on $Y$, given in the coordinate system chosen around $y_{0}$ by

$$
u \mapsto Q u(y)=\frac{1}{(2 \pi)^{n}} \iint e^{i(y-\tilde{y}) \cdot \eta} c(y, \tilde{y}, \eta) u(\tilde{y}) d \tilde{y} d \eta,
$$

its complete symbol $q(y, \eta) \in S^{m}$ in these coordinates has an asymptotic expansion

$$
q(y, \eta) \sim \sum_{|\alpha| \geq 0}(-i)^{|\alpha|} \partial_{\tilde{y}}^{\alpha} \partial_{\eta}^{\alpha} c(y, \tilde{y}, \eta)_{\mid \tilde{y}=y}
$$


from which $\sigma^{w}(Q)$ can then be written, modulo symbols in $S^{m-2}$, as (48)

$$
\begin{aligned}
\sigma^{w}(Q)(y, \eta) \equiv q(y, \eta)+ & \frac{i}{2} \sum_{k=1}^{n} \partial_{y_{k}} \partial_{\eta_{k}} q(y, \eta) \\
=c(y, y, \eta)- & i \sum_{j=1}^{n} \partial_{\tilde{y}_{k}} \partial_{\eta_{k}} c(y, \tilde{y}, \eta)_{\mid \tilde{y}=y} \\
& +\frac{i}{2} \sum_{k=1}^{n} \partial_{y_{k}} \partial_{\eta_{k}}[c(y, y, \eta)] \\
=c(y, y, \eta)- & i \sum_{j=1}^{n} \partial_{\tilde{y}_{k}} \partial_{\eta_{k}} c(y, \tilde{y}, \eta)_{\mid \tilde{y}=y} \\
& +\frac{i}{2} \sum_{k=1}^{n}\left(\partial_{y_{k}} \partial_{\eta_{k}} c(y, \tilde{y}, \eta)+\partial_{\tilde{y} k} \partial_{\eta_{k}} c(y, \tilde{y}, \eta)\right)_{\mid \tilde{y}=y} \\
=c(y, y, \eta) & +\frac{i}{2} \sum_{k=1}^{n}\left(\partial_{y_{k}} \partial_{\eta_{k}} c(y, \tilde{y}, \eta)\right. \\
& \left.-\partial_{\tilde{y}_{k}} \partial_{\eta_{k}} c(y, \tilde{y}, \eta)\right)_{\mid \tilde{y}=y} \quad\left(\bmod S^{m-2}\right) .
\end{aligned}
$$

Before we proceed with the final computations, we should still make some observations that allow us at this point to get a few further simplifications. First, we recall that the cut-off function $\chi$, used in the definition of $a$, was constructed so that it is equal to one in a conic neighborhood of $\left(x_{0}, y_{0}, \eta_{0}\right)$ for large $|\eta|$. Moreover, from Lemma 2 we can immediately conclude that the dependence of $c$ on $\tilde{y}$ comes exclusively from the symbol $b$ which, in turn, from (38) and (39), can be easily seen to be a function of $\tilde{y}$ only through the cut-off $\chi$ in $a$. Therefore, when $\tilde{y}=y$, $|\eta|$ is large and $(x, y, \eta /|\eta|)$ is close to $\left(x_{0}, y_{0}, \eta_{0} /\left|\eta_{0}\right|\right)$, the cut-off function is locally constant and equal to one, so that the second term in the sum above is zero.

For the statement of Theorem 1 we are only concerned with obtaining $\sigma^{w}(Q)(y, \eta)$ in a small conic neighborhood of $\left(y_{0}, \eta_{0}\right)$, for large $|\eta|$, for which $x=x(y, \eta)$ is close to $x_{0}$. According to the previous remarks, in this region (48) can then be simplified to

(49) $\sigma^{w}(Q)(y, \eta) \equiv c(y, y, \eta)+\frac{i}{2} \sum_{k=1}^{n} \partial_{y_{k}} \partial_{\eta_{k}} c(y, \tilde{y}, \eta)_{\mid \tilde{y}=y} \quad\left(\bmod S^{m-2}\right)$. 
Because we are ignoring differences in $S^{m-2}$, it is clear that the whole expansion for $b$ from (38) is only needed in (41) for the term $C_{0,0}$. The terms $C_{1,0}, C_{1,1}$ and $C_{1,0}$, which are already in $S^{m-1}$, require only the consideration of the leading order term $p\left(x, S_{x}^{\prime}(x, \eta)\right) a(x, \tilde{y}, \eta)$ from $(38)$, as far as their dependence on the symbol $b$ is concerned.

Following this observation, the first term $c(y, y, \eta)$ in (49), modulo symbols in $S^{m-2}$, is then given by

(50)

$$
\begin{aligned}
& c(y, y, \eta) \equiv \frac{p a^{2}}{\left|\operatorname{det} \frac{\partial^{2} S}{\partial x \partial \eta}(x, \eta)\right|}+\frac{i}{2\left|\operatorname{det} \frac{\partial^{2} S}{\partial x \partial \eta}(x, \eta)\right|} \\
& \times\left[-\sum_{k=1}^{n} \partial_{x_{k}}\left(a^{2}\right) \partial_{\xi_{k}} p-\frac{i}{2} \sum_{j, k=1}^{n} \frac{\partial^{2} S}{\partial x_{j} \partial x_{k}} a^{2} \partial_{\xi_{j}} \partial_{\xi_{k}} p\right. \\
& +\sum_{i, j, k, l=1}^{n} V_{i j} U_{i k}^{-1} U_{j l}^{-1} \partial_{x_{k}} \partial_{x_{l}}\left(p a^{2}\right)-\sum_{i, j=1}^{n} U_{i j}^{-1} \partial_{x_{j}}\left(p \partial_{\eta_{i}}\left(a^{2}\right)\right) \\
& +\sum_{i, j, k, l=1}^{n} U_{i j}^{-1} U_{k l}^{-1}\left(\frac{\partial^{3} S}{\partial x_{j} \partial x_{l} \partial \eta_{i}}\left(p \partial_{\eta_{k}}\left(a^{2}\right)\right)+2 \frac{\partial^{3} S}{\partial x_{j} \partial \eta_{i} \partial \eta_{k}} \partial_{x_{l}}\left(p a^{2}\right)\right. \\
& \left.+\frac{\partial^{4} S}{\partial x_{j} \partial x_{l} \partial \eta_{i} \partial \eta_{k}} p a^{2}\right) \\
& -\sum_{i, j, k, l, p, q=1}^{n} V_{i j} U_{i k}^{-1} U_{j l}^{-1} U_{p q}^{-1}\left(2 \frac{\partial^{3} S}{\partial x_{k} \partial x_{q} \partial \eta_{p}} \partial_{x_{l}}\left(p a^{2}\right)\right. \\
& \left.+\frac{\partial^{3} S}{\partial x_{k} \partial x_{l} \partial \eta_{p}} \partial_{x_{q}}\left(p a^{2}\right)+\frac{\partial^{4} S}{\partial x_{k} \partial x_{l} \partial x_{q} \partial \eta_{p}} p a^{2}\right) \\
& +\sum_{i, j, k, l, p, q, r, s=1}^{n} V_{i j} U_{i k}^{-1} U_{j l}^{-1} U_{p q}^{-1} U_{r s}^{-1}\left(\frac{\partial^{3} S}{\partial x_{k} \partial x_{q} \partial \eta_{p}} \frac{\partial^{3} S}{\partial x_{l} \partial x_{s} \partial \eta_{r}}\right. \\
& \left.+\frac{\partial^{3} S}{\partial x_{k} \partial x_{q} \partial \eta_{r}} \frac{\partial^{3} S}{\partial x_{l} \partial x_{s} \partial \eta_{p}}+\frac{\partial^{3} S}{\partial x_{k} \partial x_{l} \partial \eta_{p}} \frac{\partial^{3} S}{\partial x_{q} \partial x_{s} \partial \eta_{r}}\right) p a^{2} \\
& -\sum_{i, j, k, l, p, q=1}^{n} U_{i j}^{-1} U_{k l}^{-1} U_{p q}^{-1}\left(\frac{\partial^{3} S}{\partial x_{l} \partial x_{q} \partial \eta_{i}} \frac{\partial^{3} S}{\partial x_{j} \partial \eta_{p} \partial \eta_{k}}\right. \\
& \left.\left.+2 \frac{\partial^{3} S}{\partial x_{j} \partial x_{q} \partial \eta_{i}} \frac{\partial^{3} S}{\partial x_{l} \partial \eta_{k} \partial \eta_{p}}\right) p a^{2}\right] \quad\left(\bmod S^{m-2}\right) .
\end{aligned}
$$


As in Lemma 2, all the occurrences of the $x$ variable in this formula are to be evaluated at $x=x(y, \eta)$. Also note that, except for the derivatives in $\xi$ of the symbol $p$, which come from the $S^{m-1}$ terms in (38), all the other derivatives acting on $p$ that we have written above, in the $x$ variables, really mean derivatives acting on the composed function $p\left(x, S_{x}^{\prime}(x, \eta)\right)$.

At this point we make a final simplification, observing that for the $(y, \eta)$ points that we are concerned with, the cut-off function $\chi$ is equal to one and does not play any role in the previous formulas. So that, for practical purposes, the symbol $a(x, y, \eta)$ in $(39)$ can be regarded as just being equal to $\left|\operatorname{det} \frac{\partial^{2} S}{\partial x \partial \eta}(x, \eta)\right|^{1 / 2}$, when it comes to the computation of the final formulas.

From the known property that the derivative of the determinant of a nonsingular matrix $M$ is given by

$$
\partial \operatorname{det} M=\operatorname{det} M \operatorname{tr}\left(M^{-1} \partial M\right)
$$

we then have

$$
\begin{aligned}
\partial\left(a^{2}\right) & =\partial\left|\operatorname{det} \frac{\partial^{2} S}{\partial x \partial \eta}(x, \eta)\right| \\
& =\left|\operatorname{det} \frac{\partial^{2} S}{\partial x \partial \eta}(x, \eta)\right| \sum_{i, j=1}^{n}\left(\frac{\partial^{2} S}{\partial x \partial \eta}(x, \eta)\right)_{j i}^{-1} \partial \frac{\partial^{2} S}{\partial x_{i} \partial \eta_{j}}(x, \eta),
\end{aligned}
$$

where the derivative $\partial$ can be considered to be any of the partial derivatives, in the $x$ or $\eta$ variables. A second derivative applied to $a^{2}$ requires an extra formula for the derivative of the inverse of $\left(\frac{\partial^{2} S}{\partial x \partial \eta}\right)$, which can easily be obtained by differentiating the equation

$$
\sum_{j=1}^{n}\left(\frac{\partial^{2} S}{\partial x \partial \eta}(x, \eta)\right)_{i j}^{-1} \frac{\partial^{2} S}{\partial x_{j} \partial \eta_{k}}(x, \eta)=\delta_{i k} .
$$

This yields

$\partial\left(\frac{\partial^{2} S}{\partial x \partial \eta}(x, \eta)\right)_{i j}^{-1}=-\sum_{k, l=1}^{n}\left(\frac{\partial^{2} S}{\partial x \partial \eta}(x, \eta)\right)_{i k}^{-1}\left(\frac{\partial^{2} S}{\partial x \partial \eta}(x, \eta)\right)_{l j}^{-1} \partial \frac{\partial^{2} S}{\partial x_{k} \partial \eta_{l}}(x, \eta)$,

or, once $x$ is evaluated at $x=x(y, \eta)$,

$$
\partial\left(\frac{\partial^{2} S}{\partial x \partial \eta}\right)_{i j \mid x=x(y, \eta)}^{-1}=-\left.\sum_{k, l=1}^{n} U_{i k}^{-1} U_{l j}^{-1} \partial \frac{\partial^{2} S}{\partial x_{k} \partial \eta_{l}}\right|_{x=x(y, \eta)} .
$$

We now use these facts to simplify (50), leading to the mutual cancellation of all the terms that do not involve derivatives in $p$, except for the 
first one. Note that the points $\left(x, S_{x}^{\prime}(y, \eta)\right)$ at which $p$ and its derivatives are evaluated in $(50)$, become

$$
\left(x, S_{x}^{\prime}(x, \eta)\right)=(x(y, \eta), \xi(y, \eta))=\Phi(y, \eta),
$$

once $x$ is evaluated at $x(y, \eta)$. In particular, $p\left(x, S_{x}^{\prime}(x, \eta)\right)$ becomes

$$
p\left(x(y, \eta), S_{x}^{\prime}(x(y, \eta), \eta)\right)=p(\Phi(y, \eta)) .
$$

From these considerations, we then obtain the final form of (50), for $(y, \eta)$ in a small conic neighborhood of $\left(y_{0}, \eta_{0}\right)$ and large $|\eta|$,

(51)

$$
\begin{aligned}
& c(y, y, \eta) \equiv p(\Phi(y, \eta)) \\
& +\frac{i}{2}\left(\sum _ { i , j , k , l , q , r = 1 } ^ { n } \left[U_{i r}^{-1} U_{k l}^{-1} \frac{\partial^{3} S}{\partial x_{l} \partial \eta_{i} \partial \eta_{k}}\right.\right. \\
& \left.-V_{i j} U_{i k}^{-1} U_{j l}^{-1} U_{q r}^{-1} \frac{\partial^{3} S}{\partial x_{k} \partial x_{l} \partial \eta_{q}}\right] \partial_{x_{r}} p(\Phi(y, \eta)) \\
& +\sum_{i, j, k, l, p, q, r=1}^{n}\left[V_{i j} U_{i k}^{-1} U_{j l}^{-1} \frac{\partial^{3} S}{\partial x_{k} \partial x_{l} \partial x_{r}}-U_{i j}^{-1} \frac{\partial^{3} S}{\partial x_{r} \partial x_{j} \partial \eta_{i}}\right. \\
& +U_{i j}^{-1} U_{k l}^{-1} \frac{\partial^{3} S}{\partial x_{l} \partial \eta_{i} \partial \eta_{k}} \frac{\partial^{2} S}{\partial x_{j} \partial x_{r}} \\
& \left.-V_{i j} U_{i k}^{-1} U_{j l}^{-1} U_{p q}^{-1} \frac{\partial^{3} S}{\partial x_{k} \partial x_{l} \partial \eta_{p}} \frac{\partial^{2} S}{\partial x_{q} \partial x_{r}}\right] \partial_{\xi_{r}} p(\Phi(y, \eta)) \\
& +\sum_{i, j, r, s=1}^{n}\left[V_{i j} U_{i r}^{-1} U_{j s}^{-1}\right] \partial_{x_{r}} \partial_{x_{s}} p(\Phi(y, \eta)) \\
& +\sum_{i, j, k, r, s=1}^{n}\left[2 V_{i j} U_{i r}^{-1} U_{j k}^{-1} \frac{\partial^{2} S}{\partial x_{k} \partial x_{s}}\right] \partial_{x_{r}} \partial_{\xi_{s}} p(\Phi(y, \eta)) \\
& +\sum_{i, j, k, l, r, s=1}^{n}\left[V_{i j} U_{i k}^{-1} U_{j l}^{-1} \frac{\partial^{2} S}{\partial x_{k} \partial x_{r}} \frac{\partial^{2} S}{\partial x_{l} \partial x_{s}}\right. \\
& \left.\left.-\frac{\partial^{2} S}{\partial x_{r} \partial x_{s}}\right] \partial_{\xi_{r}} \partial_{\xi_{s}} p(\Phi(y, \eta))\right) \quad\left(\bmod S^{m-2}\right)
\end{aligned}
$$


The conclusion of the computation of the transformation formula for $\sigma^{w}(Q)$ will now follow once we explicitly determine the second term in $(49)$. In the $(y, \eta)$ region that we are interested in, and disregarding symbols in $S^{m-2}$, it is given by

$$
\frac{i}{2} \sum_{k=1}^{n} \partial_{y_{k}} \partial_{\eta_{k}} c(y, \tilde{y}, \eta)_{\mid \tilde{y}=y} \equiv \frac{i}{2} \sum_{k=1}^{n} \partial_{y_{k}} \partial_{\eta_{k}}[p(\Phi(y, \eta))] \quad\left(\bmod S^{m-2}\right),
$$

which can be concluded from (41), noticing that only the leading order term in $C_{0,0}(y, \tilde{y}, \eta)$ is relevant here (all the remaining terms becoming symbols in $S^{m-2}$, as a result of the derivative in the $\eta$ variable).

The derivatives of the composed symbol are easily computed

$$
\begin{aligned}
\frac{\partial^{2}}{\partial y_{k} \partial \eta_{k}} p(\Phi(y, \eta))= & \sum_{r, s=1}^{n} \frac{\partial^{2} x_{r}}{\partial y_{k} \partial \eta_{k}} \partial_{x_{r}} p(\Phi(y, \eta)) \\
& +\frac{\partial^{2} \xi_{r}}{\partial y_{k} \partial \eta_{k}} \partial_{\xi_{r}} p(\Phi(y, \eta)) \\
& +\frac{\partial x_{r}}{\partial y_{k}} \frac{\partial x_{s}}{\partial \eta_{k}} \partial_{x_{r}} \partial_{x_{s}} p(\Phi(y, \eta)) \\
& +\left(\frac{\partial x_{r}}{\partial \eta_{k}} \frac{\partial \xi_{s}}{\partial y_{k}}+\frac{\partial x_{r}}{\partial y_{k}} \frac{\partial \xi_{s}}{\partial \eta_{k}}\right) \partial_{x_{r}} \partial_{\xi_{s}} p(\Phi(y, \eta)) \\
& +\frac{\partial \xi_{r}}{\partial y_{k}} \frac{\partial \xi_{s}}{\partial \eta_{k}} \partial_{\xi_{r}} \partial_{\xi_{s}} p(\Phi(y, \eta))
\end{aligned}
$$

where, obviously, the functions $x=x(y, \eta)$ and $\xi=\xi(y, \eta)$ represent the $x$ and $\xi$ components of the canonical transformation $(x, \xi)=\Phi(y, \eta)$. Formulas for their partial derivatives can be determined, depending on the generating function $S(x, \eta)$, by differentiating the equations:

$$
\begin{aligned}
y & =S_{\eta}^{\prime}(x(y, \eta), \eta), \\
\xi(y, \eta) & =S_{x}^{\prime}(x(y, \eta), \eta),
\end{aligned}
$$

with respect to $y$ and $\eta$. From these we obtain

$$
\begin{aligned}
& \frac{\partial x_{r}}{\partial y_{k}}=U_{k r}^{-1}, \quad \frac{\partial x_{r}}{\partial \eta_{k}}=-\sum_{j=1}^{n} V_{k j} U_{j r}^{-1}, \\
& \frac{\partial \xi_{r}}{\partial y_{k}}=\sum_{j=1}^{n} U_{k j}^{-1} \frac{\partial^{2} S}{\partial x_{j} \partial x_{r}}, \quad \frac{\partial \xi_{r}}{\partial \eta_{k}}=\frac{\partial^{2} S}{\partial x_{r} \partial \eta_{k}}-\sum_{i, j=1}^{n} V_{k i} U_{i j}^{-1} \frac{\partial^{2} S}{\partial x_{j} \partial x_{r}},
\end{aligned}
$$


$\frac{\partial^{2} x_{r}}{\partial y_{k} \partial \eta_{k}}=\sum_{i, j, l, q=1}^{n}-U_{i r}^{-1} U_{k j}^{-1} \frac{\partial^{3} S}{\partial x_{j} \partial \eta_{i} \partial \eta_{k}}+V_{k j} U_{j q}^{-1} U_{k l}^{-1} U_{i r}^{-1} \frac{\partial^{3} S}{\partial x_{l} \partial x_{q} \partial \eta_{i}}$,
$\frac{\partial^{2} \xi_{r}}{\partial y_{k} \partial \eta_{k}}=\sum_{i, j, l=1}^{n} U_{k i}^{-1} \frac{\partial^{3} S}{\partial x_{i} \partial x_{r} \partial \eta_{k}}-V_{k j} U_{j i}^{-1} U_{k l}^{-1} \frac{\partial^{3} S}{\partial x_{i} \partial x_{l} \partial x_{r}}+\frac{\partial^{2} S}{\partial x_{i} \partial x_{r}} \frac{\partial^{2} x_{i}}{\partial y_{k} \partial \eta_{k}}$.

Substituting these formulas in (52) and adding the corresponding term to (51) in (49), most terms cancel out and we are left with the final formula for $\sigma^{w}(Q)$, with $(y, \eta)$ in a small conic neighborhood of $\left(y_{0}, \eta_{0}\right)$ and large $|\eta|$,

$$
\sigma^{w}(Q)(y, \eta) \equiv p(\Phi(y, \eta))+\frac{i}{2} \sum_{j=1}^{n} \partial_{x_{j}} \partial_{\xi_{j}} p(\Phi(y, \eta)) \quad\left(\bmod S^{m-2}\right)
$$

which is the formula $\sigma^{w}(Q)=\sigma^{w}(P) \circ \Phi$ that was our goal to prove.

The unitary properties of the operator $A$, given by (25) and (26), are now easy to obtain. For the first one, we make $P=I_{X}$ and apply this improved version of Egorov's theorem just proved, to conclude that $Q=A^{*} A \in \Psi^{0}\left(Y, \Omega_{Y}^{1 / 2}\right)$ has a Weyl principal symbol which, in a conic neighborhood of $\left(y_{0}, \eta_{0}\right)$, equals 1 . Therefore $Q-I_{Y} \in$ $\Psi^{-2}$ in this neighborhood. As for (26), we use the fact that $A$ is elliptic at $\left(x_{0}, \xi_{0}, y_{0}, \eta_{0}\right) \in C_{\Phi}$ to ensure the existence of a microlocal parametrix $A^{-1} \in I^{0}\left(Y \times X, C_{\Phi^{-1}} ; \Omega_{Y \times X}^{1 / 2}\right)$ in a neighborhood of that point. Following the typical argument now, we get

$$
\begin{aligned}
& A^{*} A-I_{Y} \in \Psi_{\left(y_{0}, \eta_{0}\right)}^{-2} \\
\Rightarrow & A A^{*} A-A \in I_{\left(x_{0}, \xi_{0}, y_{0}, \eta_{0}\right)}^{-2} \\
\Rightarrow & A A^{*} A A^{-1}-A A^{-1} \in \Psi_{\left(x_{0}, \xi_{0}\right)}^{-2} \\
\Rightarrow & A A^{*}-I_{X} \in \Psi_{\left(x_{0}, \xi_{0}\right)}^{-2},
\end{aligned}
$$

where the indices of the operator classes are obviously meant to indicate the points in conic neighborhoods of which the assertions are valid.

\section{References}

[BFG] M. Beals, C. Fefferman and R. Grossman, Strictly pseudoconvex domains in $C^{n}$, Bull. Amer. Math. Soc. (N.S.) 8(2) (1983), 125-322. 
[BoRo] A. Bouzouina And D. RoBert, Uniform semiclassical estimates for the propagation of quantum observables, Duke Math. J. 111(2) (2002), 223-252.

[DiSj] M. DimAssi AND J. SJÖSTRAND, "Spectral asymptotics in the semi-classical limit", London Mathematical Society Lecture Note Series 268, Cambridge University Press, Cambridge, 1999.

[Dui] J. J. DuistermaAt, "Fourier integral operators", Progress in Mathematics 130, Birkhäuser Boston, Inc., Boston, MA, 1996.

[Ego] Ju. V. EgOROV, The canonical transformations of pseudodifferential operators, (Russian), Uspehi Mat. Nauk 24 (1969), no. 5 (149), 235-236.

[Fol] G. B. Folland, "Harmonic analysis in phase space", Annals of Mathematics Studies 122, Princeton University Press, Princeton, NJ, 1989.

[GrSj] A. GRIGIS AND J. SJÖSTRAND, "Microlocal analysis for differential operators. An introduction", London Mathematical Society Lecture Note Series 196, Cambridge University Press, Cambridge, 1994.

[HeSj] B. Helffer And J. SuÖstrand, Semiclassical analysis for Harper's equation. III. Cantor structure of the spectrum, Mém. Soc. Math. France (N.S.) 39 (1989), 1-124.

[HiSj] M. Hitrik and J. SjÖstrand, Non-selfadjoint perturbations of selfadjoint operators in 2 dimensions. I, Ann. Henri Poincaré 5(1) (2004), 1-73.

[Hör1] L. HöRmANDER, The Weyl calculus of pseudodifferential operators, Comm. Pure Appl. Math. 32(3) (1979), 359-443.

[Hör2] L. HörmANDER, "The analysis of linear partial differential operators. I. Distribution theory and Fourier analysis", Second edition, Grundlehren der Mathematischen Wissenschaften 256, Springer-Verlag, Berlin, 1990.

[Hör3] L. HÖRMANDER, "The analysis of linear partial differential operators. III. Pseudo-differential operators", Corrected reprint of the 1985 original, Grundlehren der Mathematischen Wissenschaften 274, Springer-Verlag, Berlin, 1994.

[Hör4] L. HÖRMANDER, "The analysis of linear partial differential operators. IV. Fourier integral operators", Corrected reprint of the 1985 original, Grundlehren der Mathematischen Wissenschaften 275, Springer-Verlag, Berlin, 1994.

[Rob] D. RoBert, "Autour de l'approximation semi-classique", Progress in Mathematics 68, Birkhäuser Boston, Inc., Boston, MA, 1987. 
[Shu] M. A. Shubin, "Pseudodifferential operators and spectral theory", Translated from the 1978 Russian original by Stig I. Andersson, Second edition, Springer-Verlag, Berlin, 2001.

[Ste] E. M. Stein, "Harmonic analysis: real-variable methods, orthogonality, and oscillatory integrals", With the assistance of Timothy S. Murphy, Princeton Mathematical Series 43, Monographs in Harmonic Analysis III, Princeton University Press, Princeton, NJ, 1993.

[Tay] M. E. TAYLOR, "Pseudodifferential operators", Princeton Mathematical Series 34, Princeton University Press, Princeton, N.J., 1981.

[Tre] F. TRÈves, "Introduction to pseudodifferential and Fourier integral operators", 2 vols., The University Series in Mathematics. Plenum Press, New York-London, 1980.

[Wey] H. WEYL, "The theory of groups and quantum mechanics", Dover Publications, New York, 1950.

Departamento de Matemática

Instituto Superior Técnico

Av. Rovisco Pais

1049-001 Lisboa

Portugal

E-mail address: jsilva@math.ist.utl.pt 